\title{
MYC drives aggressive prostate cancer by disrupting transcriptional pause release at androgen receptor targets
}

\section{Authors}

4 Xintao Qiu ${ }^{1,2, \dagger}$, Nadia Boufaied ${ }^{3, \dagger}$, Tarek Hallal ${ }^{3,4}$, Avery Feit ${ }^{1,2}$, Anna de Polo ${ }^{3,5}$, Adrienne M. 5 Luoma $^{6}$, Janie Larocque ${ }^{3,7}$, Giorgia Zadra ${ }^{8,9}$, Yingtian Xie ${ }^{1,2}$, Shengqing Gu ${ }^{1,2,10}$, Qin Tang ${ }^{1,2,10}$, Yi 6 Zhang ${ }^{1,10}$, Sudeepa Syamala ${ }^{1}$, Ji-Heui $\mathrm{Seo}^{2}$, Connor Bell ${ }^{2}$, Edward O'Connor ${ }^{2}$, Yang Liu ${ }^{11}$, Edward 7 M. Schaeffer ${ }^{12}$, R. Jeffrey Karnes ${ }^{13}$, Sheila Weinmann ${ }^{14}$, Elai Davicioni ${ }^{11}$, Paloma Cejas ${ }^{1,2}$, Leigh 8 Ellis $^{15}$, Massimo Loda ${ }^{16}$, Kai W. Wucherpfennig ${ }^{6}$, Mark M. Pomerantz ${ }^{2}$, Daniel E. Spratt ${ }^{17}$, Eva 9 Corey $^{18}$, Matthew L. Freedman ${ }^{1,2,19}$, X. Shirley Liu ${ }^{1,10}$, Myles Brown ${ }^{1,2}$, Henry W. Long ${ }^{1,2, *}$, David 10 P. Labbé $3,4,5,7,20$,*

\section{Affiliations}

$12{ }^{1}$ Center for Functional Cancer Epigenetics, Dana-Farber Cancer Institute, Boston, MA, USA

${ }^{2}$ Department of Medical Oncology, Dana-Farber Cancer Institute, Harvard Medical School, Boston, MA, USA

${ }^{3}$ Cancer Research Program, Research Institute of the McGill University Health Centre, Montréal, Québec, Canada

${ }^{4}$ Department of Anatomy and Cell Biology, McGill University, Montréal, Québec, Canada

${ }^{5}$ Division of Urology, Department of Surgery, McGill University, Montréal, Québec, Canada

${ }^{6}$ Department of Cancer Immunology and Virology, Dana-Farber Cancer Institute, Harvard Medical School, Boston, MA, USA

${ }^{7}$ Division of Experimental Medicine, Department of Medicine, McGill University, Montréal, Québec, Canada

${ }^{8}$ Departments of Oncologic Pathology and Pathology, Dana-Farber Cancer Institute and Brigham's Women Hospital, Boston, MA, USA

${ }^{9}$ Institute of Molecular Genetics, Research National Council, Pavia, Italy

${ }^{10}$ Department of Data Science, Dana-Farber Cancer Institute, Harvard T.H. Chan School of Public Health, Boston, MA, USA

${ }^{11}$ Decipher Biosciences, San Diego, CA, USA

${ }^{12}$ Department of Urology, Northwestern University, Chicago, IL, USA

${ }^{13}$ Department of Urology, Mayo Clinic, Rochester, MN, USA

${ }^{14}$ Center for Health Research, Kaiser Permanente Northwest, Portland, OR, USA

${ }^{15}$ Division of Medical Oncology, Department of Medicine, Cedars-Sinai Medical Center and Cedars-Sinai Samual Oschin Comprehensive Cancer Institute, Los Angeles, CA, USA

${ }^{16}$ Department of Pathology and Laboratory Medicine, Weil Cornell Medicine, New York Presbyterian-Weill Cornell Campus, New York, NY, USA

${ }^{17}$ Department of Radiation Oncology, University of Michigan, Ann Arbor, MI, USA

${ }^{18}$ Department of Urology, University of Washington, Seattle, WA, USA

${ }^{19}$ The Eli and Edythe L. Broad Institute, Cambridge, MA, USA

${ }^{20}$ Lead Contact

${ }^{\dagger}$ Contributed equally

40

${ }^{*}$ Correspondence: henry_long@dfci.harvard.edu (H.W.L.), david.labbe@mcgill.ca (D.P.L.) 


\section{RUNNING TITLE}

43 MYC pauses AR transcriptional program

\section{ABSTRACT}

45 c-MYC (MYC) is a major driver of prostate cancer tumorigenesis and progression. Although MYC

46 is overexpressed in both early and metastatic disease and associated with poor survival, its impact

47 on prostate transcriptional reprogramming remains elusive. We demonstrate that MYC

48 overexpression significantly diminishes the androgen receptor (AR) transcriptional program (the

49 set of genes directly targeted by the AR protein) in luminal prostate cells without altering AR

50 expression. Importantly, analyses of clinical specimens revealed that concurrent low AR and high

51 MYC transcriptional programs accelerate prostate cancer progression toward a metastatic,

52 castration-resistant disease. Data integration of single-cell transcriptomics together with ChIP-seq

53 revealed an increased RNA polymerase II (Pol II) promoter-proximal pausing at AR-dependent

54 genes following MYC overexpression without an accompanying deactivation of AR-bound

55 enhancers. Altogether, our findings suggest that MYC overexpression antagonizes the canonical

56 AR transcriptional program and contributes to prostate tumor initiation and progression by

57 disrupting transcriptional pause release at AR-regulated genes.

\section{STATEMENT OF SIGNIFICANCE}

$59 \mathrm{AR}$ and MYC are key to prostate cancer etiology but our current understanding of their interplay

60 is scarce. Here we show that the oncogenic transcription factor MYC can pause the transcriptional

61 program of the master transcription factor in prostate cancer, AR, while turning on its own, even

62 more lethal program. 


\section{INTRODUCTION}

Prostate cancer is the most common non-cutaneous malignancy and a leading cause of cancer-

65 related lethality in men ${ }^{1}$. The androgen receptor (AR), a ligand-activated transcription factor, is central to the homeostasis of normal prostate epithelium ${ }^{2,3}$. Importantly, since the discovery that prostate cancer is reliant on androgen signaling to thrive ${ }^{4,5}$, targeting AR activity continues to be the main pillar of prostate cancer therapy ${ }^{6}$.

Prostate cancer initiation and progression involves the corruption of the normal prostate cancer transcriptional network ${ }^{7}$. Loss of the $N K X 3-1$ homeobox gene is a frequent and early event in prostate cancer etiology while the TMPRSS2-ERG gene fusion and FOXA1 mutations both identify major molecular subtypes of the disease ${ }^{8,9}$.

Overexpression of c-Myc (MYC), a master transcription factor and oncoprotein whose expression and function are tightly controlled under normal circumstances, is frequently observed in prostate cancer. Nuclear overexpression of MYC protein is an early event observed in luminal cells of prostate intraepithelial neoplasia (PIN) and is maintained in a large proportion of primary carcinomas and metastatic disease ${ }^{10}$. Importantly, about $25 \%$ of familial risk of prostate cancer map to germline variation at chromosome $8 \mathrm{q} 24$ with mechanistic evidence tying this region to $M Y C$ regulation ${ }^{11-13}$. Critically, MYC overexpression in normal luminal cells of murine prostate is sufficient to initiate prostate cancer ${ }^{14}$, providing evidence that deregulation of MYC protein expression is a critical oncogenic event driving prostate cancer initiation.

Although AR and MYC are both central to prostate cancer etiology, our current understanding of the interplay between these two transcription factors is scarce. A recent study revealed that MYC overexpression antagonizes androgen-induced gene expression in an androgen-sensitive cell line representative of advanced prostate cancer ${ }^{15}$. However, it remains unknown how increased MYC expression shapes the AR transcriptional program in normal luminal prostate cells as they transition to PIN and subsequently progress from a localized to a metastatic disease.

Here we model MYC-driven prostate cancer initiation in vivo and define the transcriptional rewiring occurring in luminal cells at a single-cell level. We demonstrate that MYC overexpression diminishes the canonical AR transcriptional program, alters the AR cistrome, and results in the establishment of a corrupted AR transcriptional program in a murine model. We determine that an active MYC transcriptional program and low AR activity identify prostate cancer patients predisposed to fail standard-of-care therapies and most likely to develop metastatic castration resistant prostate cancer (mCRPC). Accordingly, we found that high MYC mRNA expression in castration-resistant tumors is also associated with a weakened canonical AR transcriptional program and a repurposing of the AR cistrome. Critically, integration of transcriptomic and epigenomic data reveals that MYC overexpression does not lead to the deactivation of AR-bound enhancers but instead results in RNA polymerase II (Pol II) promoter-proximal pausing at ARdependent genes. Altogether, our findings suggest that MYC overexpression contributes to tumor initiation and progression by disrupting the AR transcriptional program. 


\section{RESULTS}

102 MYC induces a profound transcriptional reprogramming in murine prostate lobes

103 To examine the transcriptional reprogramming associated with MYC-driven prostate cancer 104 initiation, we compared a 12-week-old mouse that overexpresses an $\mathrm{ARR}_{2} \mathrm{~Pb}$ driven human $c-M Y C$ transgene $(M Y C)$ in the prostate epithelium to a wild-type (WT) littermate ${ }^{14}$. At 12 weeks of age, MYC overexpression induces cellular epithelium transformation to PIN, a premalignant condition that often precedes the development of invasive adenocarcinoma in humans ${ }^{16}$, with varying penetrance across prostate lobes. Notably, the murine anterior prostate (AP) remained mostly unaffected by MYC overexpression while PIN penetrance reached $83 \%$ and $97 \%$ in the dorsolateral prostate (DLP) and ventral prostate (VP), respectively ${ }^{17}$. Transcriptional profiling of whole prostate lobes at a single-cell level revealed a strong overlap with the matched bulk gene expression profiling across lobes and genotypes (WT and MYC; Figure 1A-B and Supplementary Figure S1A). Comparison of gene expression levels quantified by single-cell RNA-seq (scRNA-seq; aggregate expression) or bulk RNA-seq revealed that scRNA-seq quantitatively recapitulates bulk gene expression (Figure 1C and Supplementary Figure S1B). Accordingly, with the exception of the AP, unsupervised clustering revealed a strong correlation between single-cell transcriptome and the matched bulk transcriptome (Figure 1D) and revealed that MYC induces a profound transcriptional reprogramming in both the DLP and VP lobes (Figure 1E).

\section{Single-cell transcriptome delineates inter- and intra-prostate lobe heterogeneity}

To determine key differences between murine prostate lobes, we projected the single-cell transcriptome data into the t-distributed stochastic neighbor embedding (tSNE) space. Using known markers (Supplementary Figure S2A-B), we identified nine major subpopulations of cells across prostate lobes (Figure 1F). Notably, basal cells $\left(K r t 5^{+}, K r t 14^{\mathrm{Hi}}\right)$ were the most abundant epithelial cell subtype observed in the AP and DLP lobes, whereas luminal cells $\left(K r t 8^{\mathrm{Hi}}, K r t 18^{\mathrm{Hi}}\right)$ were overwhelmingly represented in the VP lobe. While murine $M y c(m m 10 M y c)$ was expressed across all subpopulations and prostate lobes (Supplementary Figure S2C and S3), human $c-M Y C$ transgene expression ( $h g 19 M Y C$ ) was largely restricted to the luminal subpopulation (Figure 1G) and more prevalent in the VP lobe (Figure 1H), a feature in line with the greater penetrance of the MYC-driven PIN transformation observed in the VP lobe (Figure 1I) ${ }^{17}$.

The high representation of luminal cells coupled with a robust and uniform MYC-driven PIN transition in the VP enabled us to further define distinct luminal subpopulations. K-means clustering revealed a luminal subpopulation $\left(K r t 8^{\mathrm{Hi}}, K r t 18^{\mathrm{Hi}}\right)$ common to both WT and MYC genotypes and characterized by high expression of $K r t 4$ but negative for $N k x 3-1$ expression $\left(K r t 4^{\mathrm{Hi}}\right.$, $N k x 3-1^{-}$; Figure 2A-B and Supplementary Figure S4A). Concurrent high expression of Cd44, Tacstd2 (Trop2) and Psca suggests that this subpopulation corresponds to luminal progenitor cells 18. In untransformed VP, the main luminal cell cluster was composed of two subpopulations characterized by either high or low expression of androgen-responsive genes such as Pbsn and $M s m b$ (Supplementary Figure S4B) ${ }^{19,20}$. Human $M Y C$ was predominately expressed in luminal cells (Figure 2C-D), resulting in an extensive transcriptional reprogramming within the luminal overexpressing luminal cells was identifiable even without inclusion of the human $M Y C$ transcript in the generation of the tSNE plot (Supplementary Figure S5). In agreement with MYC function in controlling transcriptional programs that favor cell growth and proliferation ${ }^{21}$, we identified a subset of highly proliferative human $M Y C$ overexpressing luminal cells positive for cyclin B1,

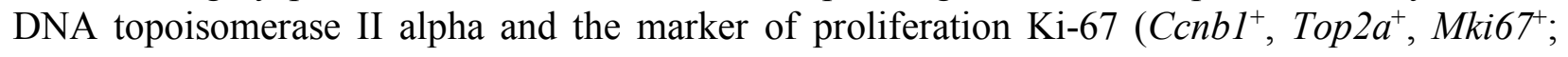


Figure 2B and Supplementary Figure S4C), a state that was independent of human or murine $M Y C$ transcript levels (Figure 2D). Finally, a limited number of cells belonging to hematopoietic $\left(\mathrm{Ptprc}^{+}\right)$, vascular endothelium $\left(\mathrm{Pdgfra}^{+}\right)$, smooth muscle $\left(\mathrm{Actg}^{+}\right)$and adipocyte $\left(\mathrm{Fabp}^{+}\right)$ populations were also identified (Figure 2B and Supplementary Figure S4D). Taken together, these results demonstrate that MYC-driven transcriptional reprogramming can be readily captured by single-cell transcriptomics.

\section{MYC-driven luminal cells transformation dampens the AR transcriptional program}

To define the transcriptional reprogramming driven by MYC overexpression in the VP lobe across cell subpopulations, we created a pseudobulk sample for each subpopulation and performed Gene Sets Enrichment Analyses (GSEA) using the Hallmark gene sets ${ }^{22}$. As expected, the pseudobulk RNA-seq analysis showed that the MYC-driven transcriptional program enriched in gene sets related to cell proliferation (E2F_targets, G2M_chekpoint) or MYC-transcriptional activity per se (MYC_targets_V1/V2), was solely driven by the luminal cells (Figure 3A-B). In fact, the near totality of the MYC-driven transcriptional program captured by bulk RNA-seq is in line with the luminal cells transcriptional program. However, a large proportion of MYC-driven transcriptional reprogramming was undetected in bulk RNA-seq and only captured by single-cell transcriptomics. Notably, basal cells underwent an extensive transcriptional reprogramming (Figure 3A). Considering that human $M Y C$ transgene expression was detected in only a limited proportion of basal cells (18.3\%; Figure 2C), this result suggests the existence of a paracrine transcriptional reprogramming upon MYC overexpression and prostate transformation. In addition, scRNA-seq revealed the downregulation of several transcriptional programs in luminal cells. Critically, the depletion of the Androgen_response gene set (Figure 3A, C), which was not accompanied with a global decreased in AR transcript and protein levels (Figure 3D-E; Supplementary Figure S4E and Supplementary Data 1), suggests a dampening of the AR transcriptional program driven by MYC overexpression as exemplified by loss of Pbsn and Msmb expression in the luminal compartment (Supplementary Figure S4B) ${ }^{19,20}$.

Thus, we sought to leverage single-cell transcriptomics to determine if $M Y C$ overexpression alters the nature of the transcripts co-expressed with $A r$ through a covariance analysis (Figure 3F $)^{23}$. As expected, androgen-dependent genes such as Pbsn, Msmb, Sbp, Defb50 and $B 2 m$ or the prostatespecific 9530002B09Rik were co-expressed with $A r$ in WT luminal cells (Figure 3G) ) $^{19,20,24-28}$. Interestingly, both Spink1 and Malat1, which are respectively associated with castration-resistant or enzalutamide-resistant disease ${ }^{29,30}$, were strongly co-expressed with $A r$ only in untransformed tissues (Figure 3G), suggesting that these genes are also part of the normal androgen-dependent prostate epithelium homeostasis. Surprisingly, upon $M Y C$ overexpression, canonical AR target genes were no longer co-expressed with $A r$. Instead, transcripts related to ribosome biogenesis, a key pathway driving cell growth and tumorigenesis and associated with MYC function ${ }^{31}$, were coexpressed with $\operatorname{Ar}$ (Figure 3G). Altogether, these results indicate that AR-transcriptional program is compromised upon MYC overexpression.

\section{MYC overexpression alters the AR cistrome}

To further characterize the mechanism whereby MYC overexpression negatively affects the ARdependent transcriptional program, we utilized chromatin immunoprecipitation followed by highthroughput sequencing (ChIP-seq) to assess the AR cistrome. Although motif analysis of AR binding sites revealed the canonical androgen response element as the top enriched motif across genotypes (Figure 4A), unsupervised clustering uncovered a distinct AR cistrome driven by MYC 190 
the AR cistrome with 1,695 sites gained compared to WT tissues (Figure 4C). Motif analyses revealed that AR gained sites are predominantly associated with the forkhead family of transcription factors motifs (forkhead response elements; FHRE), which includes the established regulator of AR transcriptional activity FOXA1, followed by androgen response elements (ARE; Figure 4D $)^{32}$. Critically, FOXA1 occupancy was increased at AR gained binding sites in MYCtransformed prostate tissues compared to the WT counterpart $(P=2.23 \mathrm{e}-62$; Figure $4 \mathbf{E}$ and Supplementary Figure S6A). Genomic regions gaining AR occupancy were characterized by increased histone H3K27 acetylation (H3K27ac; $P=4.39 \mathrm{e}-40$; Figure 4F), a mark of active regulatory regions and transcriptional activity ${ }^{33}$, supporting a differential usage of non-coding regulatory elements driven by $\mathrm{AR}$ in a $\mathrm{MYC}$ overexpressing context. To determine whether the repurposing of the $\mathrm{AR}$ cistrome upon MYC overexpression is associated with a distinct transcriptional program, we next integrated AR ChIP-seq to single-cell transcriptomics. Association of 1,695 AR binding sites gained upon MYC overexpression (Figure 4C) to the expression of nearby coding genes in the luminal cell subpopulations, ordered based on slingshot pseudotime inference across genotypes (Supplementary Figure S6B), highlighted three main expression patterns, namely a MYC-dependent increased, decreased or unchanged expression (Figure 4G). Using GSEA analysis and the Hallmark gene sets, we identified the MYC_targets_V1 as the top gene set enriched within the set of genes with increased expression. Conversely, we identified the Androgen_response among the gene sets that were significantly enriched within the set of genes with decreased expression (Figure 4H). Taken together these results indicate that, in the context of MYC overexpression, a reprogramming of the AR cistrome that drives an altered transcriptional program.

\section{Divergent MYC and AR transcriptional programs dictate disease progression}

214

Since our results in the preclinical model uncovered a robust interplay between MYC and AR transcriptional programs, we next investigated whether this MYC-driven transcriptional reprogramming is clinically relevant. We used gene expression data to stratify 488 primary prostate cancer patients in the TCGA dataset based on the combined levels of the Hallmark Androgen_response (high; low) and MYC_targets_V1 (high; low) transcriptional signatures ${ }^{9}$. Kaplan-Meier curves revealed that patients bearing a primary tumor characterized by divergent AR and MYC transcriptional programs experienced distinct rates of clinical progression. Tumors characterized by a low AR transcriptional signature with concurrent high MYC transcriptional signature (AR_low/MYC_high) were associated with the shortest time to biochemical recurrence (BCR) while tumors characterized by a high AR transcriptional signature with concurrent low MYC transcriptional signature (AR_high/MYC_low) were associated with the longest time to BCR (Supplementary Figure S7A-B). Interestingly, concordant AR and MYC transcriptional programs (AR_high/MYC_high; AR_low/MYC_low) were associated with an intermediate time to BCR

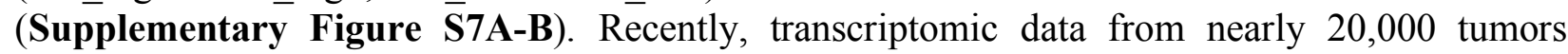
revealed that patients bearing a localized treatment-naïve primary prostate cancer with low ARactivity (AR-A; based on a signature of nine canonical AR transcriptional targets) experience a shorter time to recurrence ${ }^{34}$. Thus, we next sought to determine if MYC transcriptional activity status in low AR-A tumors could identify a more aggressive subtype of primary prostate cancer using the TCGA dataset. Strikingly, Kaplan-Meier curves revealed that it is the subset of low ARA tumors with concurrent high MYC transcriptional signature that is associated with a faster time to BCR (AR_low/MYC_high vs. AR_low/MYC_low, $P=0.0001$; Figure 5A-B). Importantly, we validated this finding in a previously published independent meta-analysis cohort combining 855 
Univariable analysis revealed that tumors with AR_low/MYC_high transcriptional signatures are associated with increased rates of BCR (Hazard Ratio (HR) $=1.37,95 \%$ Confidence Interval (CI) 1.03-1.83; $P=0.030$; Figure 5D), but this did not remain significant after adjusting for clinicopathologic risk factors in multivariable analysis (Figure 5D and Supplementary Figure S7D). Since low AR-A tumors were predicted to be less sensitive to androgen-deprivation therapy and more likely to develop metastatic disease after initial local therapy ${ }^{34}$, we next asked whether a high MYC transcriptional activity allows for the identification of a more aggressive subtype of treatment-naïve primary prostate cancer. Strikingly, Kaplan-Meir curves revealed that patients with tumors harboring an AR_low/MYC_high signature were the most likely to develop metastatic disease (Figure 5E and Supplementary Figure S7E). Univariable analysis shows that AR_low/MYC_high tumors are associated with an increased risk to develop metastatic disease $\left(\mathrm{HR}^{-}=2.93,95 \%\right.$ CI 1.68-5.10; $P<0.001$; Figure 5F and Supplementary Figure S7F $)$. Critically, this finding remained significant in a multivariable competing risks regression analysis adjusting for age, prostate-specific antigen (PSA), Gleason score, surgical margin status, extracapsular extension, seminal vesicles invasion and lymph node involvement $(\mathrm{HR}=2.46,95 \%$ CI $1.34-4.52$; $P=0.004$; Figure 5F and Supplementary Figure S7F). Altogether, our results suggest that concurrent AR_low/MYC_high transcriptional signatures identify a subgroup of patients that are predisposed to fail standard-of-care therapies and progress to develop metastatic disease.

High MYC expression is associated with a dampened AR transcriptional program and an expanded AR cistrome in castration-resistant tumors

CRPC is characterized by MYC and AR amplification ${ }^{9,15,36}$. Thus, we sought to assess the impact of MYC expression on the AR transcriptional program and cistrome. Gene expression profiling from $59 \mathrm{AR}^{+} \mathrm{CRPC}$ tumors revealed that $\mathrm{AR}$ activity is negatively correlated with $M Y C$ expression (Figure 6A-B $)^{37}$. As expected, GSEA analysis revealed that $M Y C$-high CRPC tumors are enriched for MYC transcriptional signatures. Strikingly, the Hallmark Androgen_response was the only gene set significantly depleted in MYC-high tumors (Figure 6C), supporting a role for MYC in dampening the canonical AR transcriptional program in the castration-resistant setting. We next determined whether this phenotype was associated with a repurposing of the AR cistrome using the $\mathrm{LuCaP}$ patient-derived xenografts (PDXs) series obtained from $\mathrm{AR}^{+} \mathrm{mCRPC}$ samples (described in ${ }^{38}$ and Supplementary Figure S8A). We selected eight specimens, for which the gene expression profiles were readily available, and stratified them into either the MYC-high or the $M Y C$-low group based on transcript expression (Figure 6D) ${ }^{37}$. Importantly, AR transcript level was not different between the MYC-high and MYC-low groups (Figure 6D). Comparison of the AR cistrome between the two groups uncovered an alteration of AR binding in $M Y C$-high mCRPC PDXs towards an expanded AR cistrome robustly associated with the forkhead family of transcription factors motifs (Figure 6E-F, Supplementary Figure S8B). Accordingly, greater FOXA1 occupancy was observed at AR gained binding sites in $M Y C$-high compared to the $M Y C$ low mCRPC PDXs ( $P=1.74 \mathrm{e}-144$; Figure 6G and Supplementary Figure S8C). These sites were also characterized by increased H3K27ac mark $(P=3.54 \mathrm{e}-268$; Figure $6 \mathrm{H})$, in agreement with the MYC-driven murine prostate cancer model (Figure 4). Critically, differential AR chromatin occupancy between both groups was associated with a dampened AR transcriptional program in the $M Y C$-high group (Figure 6I). Taken together, these results support the existence of a distinct AR cistrome in MYC overexpressing CRPC associated with a diminished AR transcriptional program. 


\section{MYC overexpression disrupts the AR transcriptional program by pausing AR regulated} genes

To assess for direct effects of AR in mediating this transcriptional reprogramming we leveraged the preclinical model of MYC-driven prostate cancer and performed binding and expression target analysis (BETA) to integrate MYC-driven gene expression changes in murine VP with genomewide AR binding data ${ }^{39}$. This analysis revealed that AR binding was significantly associated with genes downregulated by MYC overexpression $(P=2.32 \mathrm{e}-5$; Figure 7A). Along this line, AR binding was found to be increased at genomic regions nearby Androgen_response genes alongside the H3K27ac mark following MYC overexpression (Figure 7B-C), in contrast with the accompanied depletion of the Androgen_response gene set (Figure 3C). For example, AR and FOXA1 binding was increased in the promoter region of Pbsn (Figure 7D), an AR-dependent gene whose transcript and protein levels were both severely downregulated following MYC overexpression (Figure 7E-F; Supplementary Figure S4B and Supplementary Data 2). In the promoter region of $M s m b$, another AR-dependent gene previously characterized as a tumor suppressor ${ }^{40}$, AR and FOXA1 binding as well as the H3K27ac mark levels were maintained although Msmb transcript levels were also downregulated by MYC overexpression (Figure 7G-H and Supplementary Figure S4B). These results suggest that MYC-driven repression of the AR transcriptional program is not associated with a disengagement of AR or the loss of the H3K27ac mark.

Using the androgen responsive $\mathrm{LNCaP}$ prostate cancer cell line, Barfeld and colleagues have previously reported that MYC overexpression antagonizes the transcriptional activity of the AR ${ }^{15}$. Similarly to the MYC-driven genetically engineered prostate cancer mouse model, MYC overexpression in LNCaP cells was associated with the depletion of the Hallmark Androgen_response gene set (Supplementary Figure S9A). Annotation of the AR cistrome and gene expression data by BETA revealed that AR binding is associated with downregulated genes, supporting a global reduction in AR transcriptional activity driven by MYC overexpression. Conversely, MYC cistrome was predominantly associated with upregulated genes, consistent with its role as a transcriptional activator (Supplementary Figure S9B). Again, AR binding nearby Androgen_response genes remained largely unchanged following MYC overexpression. Interestingly, MYC binding nearby MYC_targets_V1 genes also remained unchanged following MYC overexpression despite a significant enrichment of the MYC_targets_V1 gene set (Supplementary Figure S9C). Inspection of AR and MYC binding in the vicinity of canonical AR-dependent genes such as KLK3 and TMPRSS2 also revealed unchanged binding profiles (Supplementary Figure S9D).

Based on the evidence for MYC regulation of RNA Pol II pause release ${ }^{41}$, we leveraged RNA Pol II ChIP-seq to determine genome-wide RNA Pol II traveling ratio (i.e. RNA Pol II density in the promoter-proximal region over the RNA Pol II density in the transcribed region) in vivo following MYC overexpression in murine VP (Figure 7I). As expected, genes with reduced RNA Pol II traveling ratio following MYC overexpression were enriched for MYC transcriptional signatures, indicative of pause release at these sites (Figure 7J-K and Supplementary Figure S10A). Critically, genes with greater RNA Pol II traveling ratio were enriched for the AR transcriptional signature, suggestive of enhanced RNA Pol II pausing at AR-regulated genes (Figure 7L-M and Supplementary Figure S10A). Along this line, ChIP-seq revealed a build-up of RNA Pol II occupancy at the promoter of the AR-regulated gene Pbsn following MYC overexpression (Figure 70). At the Msmb locus, another AR-regulated gene, RNA Pol II occupancy remained unchanged at the promoter region but was abrogated at the gene body in the MYC overexpressing condition 
327 (Figure 7P). These features are in stark contrast to MYC-regulated genes such as Rps3 and Rps5 328 for which we observed an increase RNA Pol II occupancy at the gene body in the MYC 329 overexpressing condition (Supplementary Figure S10B- C). Since these patterns suggest a MYC330 driven altered ratio of initiating and elongating RNA Pol II at AR-regulated genes, we next 331 determined the RNA Pol II traveling ratio at Androgen_response genes. Strikingly, RNA Pol II 332 traveling ratio at Androgen_response genes was significantly increased by MYC overexpression $333(P=0.0021$; Figure 7Q and Supplementary Figure S10D), supporting MYC-driven RNA Pol II 334 promoter-proximal pausing and consequently non-productive transcription at AR-dependent genes. 335 Altogether these findings support RNA Pol II promoter-proximal pausing as a potential mechanism 336 for MYC-mediated transcriptional repression at AR regulated genes associated with the canonical 337 AR transcriptional signature (Graphical Summary) $)^{42}$. 


\section{DISCUSSION}

339 In this study, we report the impact of MYC overexpression in vivo on the AR transcriptional 340 program. By leveraging the expression of a human MYC transgene (hg19MYC) observed at a 341 single-cell level in murine prostatic tissues, our data demonstrate that MYC overexpression robustly reprograms luminal $\left(K r t 8^{\mathrm{Hi}}, K r t 18^{\mathrm{Hi}}\right)$ cells toward a repressed AR transcriptional program, a feature contrasting with the supporting role of MYC on the AR transcriptional program in the apocrine breast cancer subtype ${ }^{43}$. Our single-cell transcriptome data delineate a minor luminal subpopulation expressing high levels of Cd44, Tacstd2 (Trop2) and Psca markers associated with luminal progenitor cells ${ }^{18}$. Recently, single-cell transcriptomics performed in the murine AP lobe also revealed a distinct but rare luminal subpopulation anatomically lining the proximal duct and expressing Tacstd2 (Trop2), Psca as well as Ly6a (Sca-1), Krt4 and Cldn10 ${ }^{44}$. An independent study suggested that the luminal subpopulation expressing high levels of progenitor markers such as Tacstd2 (Trop2), Psca, Ly6a (Sca-1) and Krt4 corresponds to urethral luminal cells extending into the proximal ducts of the prostate ${ }^{45}$. Since the luminal progenitor population identified in the VP lobe expressed all the aforementioned markers (Supplementary Figure S4A, F), we cannot rule out the possibility that they might be of urethral origin. Regardless, these progenitor cells were not transcriptionally reprogramed following MYC overexpression (Figure 3A).

In analyzing the expression of $h g 19 M Y C$ transcript driven by the $\mathrm{ARR}_{2} \mathrm{~Pb}$ promoter we found it was not detected in WT prostates, as expected. Surprisingly, we detected low, but consistent hg19MYC expression in non-luminal subpopulations (basal: 17/93 (18.3\%); hematopoietic: 3/35 (8.6\%); vascular endothelium: $1 / 8(12.5 \%)$; Figure 2C). While the $\mathrm{ARR}_{2} \mathrm{~Pb}$ promoter used to drive hg19MYC expression has been described as highly specific for prostatic epithelium ${ }^{14,20,46}$, our single-cell transcriptome highlights a potentially underappreciated leaky expression of $\mathrm{ARR}_{2} \mathrm{~Pb}$ driven transgene. However, these seemingly stochastic events are likely transient since Hi-MYC mice do not develop other MYC-driven malignancies, such as B-cell leukemia/lymphoma ${ }^{47}$. With the increasing availability of single-cell transcriptomic profiles from various genetically engineered mouse models (GEMMs), it is expected that tissue specific promoter specificity will be reassessed through a new lens.

MYC is commonly amplified in primary prostate cancer and is overexpressed in $37 \%$ of metastatic disease 9,48 . Considering that prostate cancer cells that develop resistance to AR-targeted therapy usually maintain AR expression ${ }^{49,50}$, the interplay between MYC and AR is likely to remain critical as the disease progress to the CRPC stage. Importantly, our analyses exposed a subtype of primary prostate cancer characterized by divergent AR (low) and MYC (high) transcriptional signatures that are predisposed to fail standard-of-care therapies and progress to the mCRPC stage (Figure 5). Arriaga and colleagues have recently reported a MYC and RAS co-activation signature associated with metastatic progression and failure to anti-androgen treatments ${ }^{51}$. It is thus tempting to speculate that MYC decreases the reliance of prostate cancer cells on the canonical AR transcriptional program, therefore facilitating resistance to AR-targeted therapies. Along this line, Bai et al. recently showed that a c-Myc inhibitor disrupting c-Myc and Max dimerization sensitizes enzalutamide-resistant prostate cancer cells to growth inhibition by enzalutamide ${ }^{52}$. Considering that transition from CRPC to neuroendocrine prostate cancer (NEPC) is driven by N-Myc, which also abrogates AR transcriptional program, and that N-Myc is functionally complementary to cMyc in various processes ${ }^{53,54}$, it is now evident that Myc family members are key to prostate cancer etiology and resistance to standard-of-care therapies. 
Intriguingly, although MYC overexpression antagonizes the AR transcriptional program, this was not associated with a diminished but rather an expanded AR cistrome, characterized by FOXA1 384 co-occupancy and an active chromatin state. Data from our MYC-driven prostate cancer mouse model, together with a previously published $\mathrm{LNCaP}$ model engineered to overexpress $\mathrm{MYC}$, revealed that MYC-driven repression of the AR transcriptional program is not associated with a disengagement of AR or the loss of the H3K27ac mark. Rather, we observed greater RNA Pol II promoter-proximal pausing and non-productive transcription at AR-dependent genes repressed by MYC in vivo. Importantly, no evidence of direct interaction between MYC and AR has been found 15,52 , suggesting that the suppression of the AR transcriptional program is not guided by a physical interaction with MYC but rather by a MYC-induced RNA Pol II pausing overcoming the AR enhancers driving AR-regulated genes. Taken together, these results support cofactor redistribution driven by increased MYC expression and resulting in greater RNA Pol II promoter-proximal 394 pausing as a potential mechanism for MYC-mediated transcriptional repression at genes regulated specifically by the AR (Graphical Summary) ${ }^{42,55}$.

396 Altogether, our study revealed an intricate crosstalk between the AR, MYC, FOXA1 and RNA Pol II resulting in a corrupted AR transcriptional program and promoting prostate cancer initiation and progression to the $\mathrm{mCRPC}$ stage. Considering that a simple dietary intervention meant to reduce saturated fat consumption can dampen MYC transcriptional program, and the recent development of viable MYC inhibitors for therapeutic interventions ${ }^{17,56}$, we foresee that targeting MYC may help restore a canonical AR transcriptional program and sensitize prostate cancer to AR-targeted therapies. 


\section{Acknowledgements}

We thank Zach Herbert for technical assistance, Noriko Uetani for graphical summary design and drawings and Marie-Claude Gingras and Livia Garzia for critical review of this manuscript. J.L. is a recipient of a Canadian Institute of Health Research Frederick Banting and Charles Best Canada Graduate Scholarship-Master's and of a Research Institute of the McGill University Health Centre M.Sc. Studentship award. Establishment and characterization of the LuCaP PDX models has been supported by the Pacific Northwest Prostate Cancer SPORE (P50CA97186), the U.S. Department of Defense Prostate Cancer Biorepository Network (W81XWH-14-2-0183), the National Cancer Institute (P01 CA163227), the Prostate Cancer Foundation, the Institute for Prostate Cancer Research, and the Richard M. Lucas Foundation. We would like to thank the patients who Development Award from the U.S. Department of Defense (PC150263) and the Barr Award from the Dana-Farber Cancer Institute. This work has been supported by National Institutes of Health grants to K.W.W. (R01 CA238039; R01 CA251599), a Prostate Cancer Foundation Challenge Award to M.M.P. and M.L.F. and grants to M.L.F. (National Institutes of Health, R01 GM107427 Medical Research Foundation). E.C., M.B. and H.W.L. acknowledge support from the National Institutes of Health (P01 CA163227-06A1). D.P.L is a Lewis Katz - Young Investigator of the Prostate Cancer Foundation and is the recipient of a Scholarship for the Next Generation of Scientists from the Cancer Research Society and is also a Research Scholar - Junior 1 from The Fonds de Recherche du Québec - Santé. The work reported here was funded by a Canadian Institutes of Health Research project grant (PJT-162246) to D.P.L.

Author contributions

Conceptualization, X.Q., M.B., H.W.L. and D.P.L.;

Methodology: X.Q., N.B., A.F. and Y.X.;

Software: X.Q., N.B., A.F. and Y.X.;

Validation: Y.L., E.D. and D.E.S.;

Formal Analysis, X.Q., N.B., A.F., Y.X., S.G., Q.T., Y.Z. and D.P.L.;

Investigation, T.H., A.d.P., A.M.L., J.L., G.Z., S.S., J-H.S., C.B., E.O'C., P.C. and D.P.L.;

Resources, E.M.S., R.J.K., S.W., L.E., M.L., K.W.W., M.M.P., E.C., M.L.F., X.S.L., M.B., H.W.L. and D.P.L.;

Data Curation, X.Q. and N.B.;

Writing - Original Draft, D.P.L.;

Writing - Review \& Editing, X.Q., N.B., G.Z., E.C., M.F., H.W.L. and D.P.L.;

Visualization, X.Q., N.B. and D.P.L.;

Supervision, H.W.L. and D.P.L.;

Project Administration, H.W.L. and D.P.L.;

440 Funding Acquisition, M.B., H.W.L. and D.P.L.

441 Competing interests

442 S.W. receives research funding from PreludeDX. D.E.S. receives personal fees from Janssen,

443 AstraZeneca, and Blue Earth and funding from Janssen. X.S.L. is a cofounder, board member, 444 and consultant of GV20 Oncotherapy and its subsidiaries, SAB of 3DMedCare, consultant for 445 Genentech, and stockholder of BMY, TMO, WBA, ABT, ABBV, and JNJ, and receives research Bio. The remaining authors declare no competing interests. 


\section{METHODS}

\section{Animal husbandry}

451 FVB Hi-MYC mice (strain number 01XK8), expressing the human c-MYC transgene in prostatic 452 epithelium, were obtained from the National Cancer Institute Mouse Repository at Frederick National Laboratory for Cancer Research ${ }^{14}$. Upon weaning (3 weeks), male mice heterozygous for the transgene (MYC), together with their wild type littermates (WT), were fed a purified diet (TD.130838, Envigo). Animals were kept on a 12-hour light / 12-hour dark cycle, and allowed free access to food and water at the Dana-Farber Cancer Institute (DFCI) Animal Resources Facility. The animal protocol was reviewed and approved by the DFCI Institutional Care and Use Committee (IACUC), and was in accordance with the Animal Welfare Act. For protein expression experiments, mice were housed in the Animal Resources Facility at the Research Institute of the McGill University Health Centre (RI-MUHC) where they were fed a regular lab chow (T.2918, Envigo) from the time of weaning. The animal protocol followed the ethical guidelines of the Canadian Council on Animal Care, and was approved by the RI-MUHC Glen Facility Animal Care Committee (FACC).

Genotyping: Tail snips were sent to Transnetyx (Transnetyx, Inc.) for genotyping or genomic DNA was extracted from ear punches using $0.4 \mathrm{~mL}$ of lysis buffer $(100 \mathrm{mM}$ Tris-HCl pH 7.5, EDTA 5 $\mathrm{mM}, 2 \% \mathrm{SDS}, 200 \mathrm{mM} \mathrm{NaCl}$ and $100 \mu \mathrm{g} / \mu \mathrm{L}$ freshly added Proteinase K). Samples were incubated overnight at $52^{\circ} \mathrm{C}$. After centrifugation at $13,000 \mathrm{rpm}$ for 20 minutes, the supernatant was collected and mixed by inversion with $0.4 \mathrm{~mL}$ isopropanol to precipitate the DNA, which was pelleted by centrifugation for 5 minutes, then washed with $0.5 \mathrm{~mL} 70 \%$ ethanol and dissolved in $10 \mu \mathrm{L}$ molecular grade water. The presence of the MYC transgene was detected by polymerase chain reaction (PCR), using the following primer combination: primer 1: 5' AAA CAT GAT GAC TAC CAA GCT TGG C 3'and primer 2: 5' ATG ATA GCA TCT TGT TCT TAG TCT TTT TCT TAA TAG GG 3'. PCR products were resolved using a 2\% agarose tris-acetate-EDTA gel and a $177 \mathrm{bp}$ band was visualized using the ChemiDoc ${ }^{\mathrm{TM}}$ imaging system (Bio-Rad).

Tissue specimens

FVB Hi-MYC model: At 12 weeks of age, mice were euthanized by $\mathrm{CO}_{2}$ / isoflurane followed by cervical dislocation. Mouse prostate lobes (AP, DLP, VP) were dissected, weighed and immediately processed for bulk and single-cell transcriptomics or flash-frozen in liquid nitrogen for chromatin immunoprecipitation or protein expression experiments. Tissues were consistently collected during the same periods to minimize inter-samples and circadian rhythm variability.

mCRPC LuCaP PDXs: Informed consent was obtained to collect human mCRPC tissues and generate the patient-derived xenograft tumors as described previously ${ }^{37,38}$. The study was approved by the University of Washington Human Subjects Division institutional review board (no. 39053). All animal studies were approved by University of Washington IACUC and performed according to NIH guidelines. Molecular characterization of $\mathrm{AR}^{+}$mCRPC LuCaP PDXs 70CR, 78CR, 81CR, 96CR, 105CR, 136CR and 147CR was previously described ${ }^{37,38}$. LuCaP PDX 167CR was established from a liver metastasis of 77-year-old Caucasian male who died of abiraterone-, carboplatin- and docetaxel-resistant CRPC. LuCaP 167CR expresses AR, responds to castration and is negative for synaptophysin. PDX cellular morphology recapitulates the original liver metastasis (Supplementary Figure S8A).

\section{Bulk RNA-sequencing}

492 FVB Hi-MYC model: Fresh prostate lobes from 12-week-old mice were dissociated to form a 493 single cell suspension. Prostate lobes were minced with a sterile razor blade and resuspended in 
collagenase/hyaluronidase (\#07912, Stemcell Technologies) diluted in DMEM/F-12 (\#36254, Stemcell Technologies) at $37^{\circ} \mathrm{C}$ for 2 hours. After dissociation, cells were centrifuged ( $350 \mathrm{x} \mathrm{g}$ for 5 minutes) and resuspended in $5 \mathrm{~mL}$ of prewarmed $0.25 \%$ trypsin/EDTA (\#07901, Stemcell Technologies) at $37^{\circ} \mathrm{C}$ for 5 minutes. Trypsinization was stopped with $10 \mathrm{~mL}$ of cold HBSS (\#37150, Stemcell) supplemented with $2 \%$ of regular cell culture grade FBS. Cells were centrifuged (350 x g for 5 minutes) and resuspended in $1 \mathrm{~mL}$ of prewarmed dispase (\#07913, Stemcell Technologies) and $100 \mu \mathrm{L}$ of DNase I (\#07900, Stemcell Technologies) and passed 5 times through a $27 \mathrm{G}$ syringe needle. Cells were then mixed with $10 \mathrm{~mL}$ of cold HBSS supplemented with $2 \%$ FBS, filtered through a $40 \mu \mathrm{m}$ cell strainer (\#27305, Stemcell Technologies), centrifuged (350 x g for 10 minutes) and resuspended in PBS. RNA from an equal number of cells was extracted using the miRNeasy Micro Kit (\#217084, Qiagen) coupled with oncolumn DNAse treatment (\#79254, Qiagen). RNA sample concentration was measured and subjected to quality evaluation, using a Bioanalyzer RNA 6000 Nano kit (\#5067-1511, Agilent). The Dana-Farber Cancer Institute Molecular Biology Core Facilities prepared libraries from 500 ng of purified total RNA, using TruSeq Stranded mRNA sample preparation kits (\#RS-122-2101, Illumina) according to the manufacturer's protocol. Finished libraries were quantified by the Qubit dsDNA High-Sensitivity Assay Kit (\#32854, Thermo Fisher Scientific), by an Agilent TapeStation 2200 system using D1000 ScreenTape (\#5067-5582, Agilent), and by RT-qPCR using the KAPA library quantification kit (\#KK4835, Kapa Biosystems), according to the manufacturers' protocols; pooled uniquely indexed RNA-seq libraries in equimolar ratios were sequenced to a target depth of 40M reads on an Illumina NextSeq500 run with single-end 75 bp reads. Read alignment, quality control and data analysis was performed using VIPER ${ }^{57}$, RNA-seq reads were mapped by STAR 58 and read counts for each gene were generated by Cufflinks ${ }^{59}$. Differential gene expression analyses were performed on absolute gene counts for RNA-seq data and raw read counts for transcriptomic profiling data using DESeq $2{ }^{60}$. mCRPC LuCaP PDXs: LuCaP PDX tumor samples were collected from castrated CB 17 SCID male mice. Frozen tumors were used for RNA extraction and RNA-seq analysis as described previously ${ }^{37}$.

522 LNCaP MYC model: Published gene expression data (GSE73995; ${ }^{15}$ ) was downloaded and 523 reanalyzed.

\section{Single-cell RNA-sequencing}

525 Cell preparation for 3' barcoded scRNA-seq (\#120237, Chromium V2 assay) was performed according to the manufacturer's protocol (10X Genomics) targeting 5000 cells from single-cell suspensions of freshly processed prostate lobes as described above. Single-cell RNA-seq data were preprocessed using the 10x genomics Cell Ranger (https://www.10xgenomics.com/) to obtain the UMI (unique molecular identifier) counts for each gene. To get a reliable single cell transcriptome dataset, we excluded the cells with fewer than 200 genes expressed (UMI $>0$ ) or the cells with more than $80 \%$ UMIs from mitochondrial genes. The filtered data was then normalized and scaled by using Seurat to remove unwanted sources of variations ${ }^{61}$. tSNE was performed on the normalized data to visualize the single cells in two-dimensional space by using the result of principal component analysis (PCA). Unsupervised clustering was performed by using the "FindClusters" function in the Seurat package with parameters of resolution $=0.8$. Genes with calculated to correct for multiple testing. 
Specific gene expression levels: The normalized expression level for all cells was calculated by the

Seurat R package (3.1.1). The Violin plots were created by the geom_violin function in ggplot2 (3.3.2), scale option set to 'area'.

Covariance analysis: The covariance for all genes with $\mathrm{Ar}$ is calculated by the cov function in stats $\mathrm{R}$ package (3.6.0). Genes that have covariance difference larger than 30 between the WT and MYC samples were colored in red and labeled in the plot. Slingshot pseudotime inference: Pseudotime inference is done by the slingshot version 1.3.1. Kmeans clustering results and tSNE coordinates were used as input for the pseudotime inference.

\section{Bioinformatics analyses - bulk RNA-seq and scRNA-seq}

Bulk RNA-seq and scRNA-seq gene expression correlation: X-axis is the $\log$ (scRNA-seq sum of UMI from all cells), Y-axis is $\log$ (bulk RNA-seq - raw read counts). Correlation is calculated based on Pearson Correlation. The Venn diagram is the overlap expressed genes between scRNAseq and bulk RNA-seq. A gene is considered as expressed when the sum of UMI from all cells is larger than 0 in scRNA-seq or raw read counts is larger than 0 in bulk-RNA-seq.

Sample-sample correlation and principal component analysis (PCA): Sum of UMI from all cells in scRNA-seq and raw read counts in bulk RNA-seq for matched samples were calculated. Batch effects between scRNA-seq and bulk RNA-seq data were removed using the ComBat approach from SVA (3.18.00). Pearson correlation and principal components were calculated using the counts after removal of batch effect.

Gene set enrichment analysis (GSEA): All GSEA were done using pre-ranked analysis (GSEA Java; v4.1.0) with Hallmark gene sets (h.all.v7.2.symbols.gmt). Heatmap visualization of normalized enrichment score (NES) was obtained using ComplexHeatmap (2.2.0) R package ${ }^{62}$.

\section{Protein expression}

Fresh-frozen VP tissues from 12-week-old mice were sliced on ice with stainless steel disposable scalpels (Fisher Scientific) then homogenized in RIPA buffer $(20 \mathrm{mM}$ Tris- $\mathrm{HCl} \mathrm{pH} 7.5,150 \mathrm{mM}$ $\mathrm{NaCl}, 1 \mathrm{mM}$ EDTA, 1\% TRITON-X) supplemented with phosphatases and protease inhibitors (Mini, Pierce ${ }^{\mathrm{TM}}$, Thermo Fisher) using a tissue grinder kit (Kontes). Equal amounts of protein (15 $\mu \mathrm{g}$; Pierce ${ }^{\mathrm{TM}}$ Rapid Gold BCA Protein Assay, Thermo Fisher) were resolved on 8-12\% Trisglycine SDS-polyacrylamide gels and transferred to nitrocellulose blotting membranes (Bio-Rad), following standard procedures. Membranes were probed with the following antibodies according to the manufacturer's instructions: rabbit monoclonal [Y69] anti-c-MYC (\#ab32072, Abcam), rabbit monoclonal [ER179(2)] anti-AR (\#ab108341, Abcam), mouse monoclonal [F6] antiprobasin (\#sc-393830, Santa Cruz Biotechnology) or rabbit polyclonal anti- $\beta$-Actin (\#4967, Cell Signaling Technology). Densitometry analyses were made with ImageJ (U.S. NIH, Bethesda, MD;

\section{ChIP-sequencing}

574 FVB Hi-MYC model: ChIP-sequencing was performed as described in Labbé and Zadra et al. ${ }^{17}$. Briefly, fresh-frozen VP tissues from 12-week-old mice were pulverized (Cryoprep Impactor, Covaris), resuspended in PBS $+1 \%$ formaldehyde, and incubated at room temperature for 20 minutes. Fixation was stopped by the addition of $0.125 \mathrm{M}$ glycine (final concentration) for 15 minutes at room temperature, then washed with ice cold PBS + EDTA-free protease inhibitor cocktail (PIC; \#04693132001, Roche). Multiple biological replicates were combined for each condition in two distinct pools (replicates). Chromatin was isolated by the addition of lysis buffer 
were maintained on ice for 30 minutes. Lysates were sonicated (E210 Focused-ultrasonicator, Covaris) and the DNA was sheared to an average length of $\sim 200-500 \mathrm{bp}$. Genomic DNA (input) was isolated by treating sheared chromatin samples with RNase $\left(30\right.$ minutes at $\left.37^{\circ} \mathrm{C}\right)$, proteinase $\mathrm{K}\left(30\right.$ minutes at $\left.55^{\circ} \mathrm{C}\right)$, de-crosslinking buffer (1\% SDS, $100 \mathrm{mM} \mathrm{NaHCO} 3$ (final concentration), 6-16 hours at $65^{\circ} \mathrm{C}$ ), followed by purification (\#28008, Qiagen). DNA was quantified on a NanoDrop spectrophotometer, using the Quant-iT High-Sensitivity dsDNA Assay Kit (\#Q33120, Thermo Fisher Scientific). On ice, AR (2 $\mu \mathrm{g}$, \#ab108341, Abcam), FOXA1 (6 $\mu \mathrm{g}$, \#ab23738, Abcam), RNA Pol II (4 $\mu$ g, \#sc899, Santa Cruz Biotechnology) or H3K27ac (10 $\mu$ l, \#ab4729, Abcam) antibodies were conjugated to a mix of washed Dynabeads protein A and G (Thermo Fisher Scientific), and incubated on a rotator (overnight at $4^{\circ} \mathrm{C}$ ) with $5 \mu \mathrm{g}$ (AR, FOXA1, RNA Pol II) or $1.5 \mu \mathrm{g}(\mathrm{H} 3 \mathrm{~K} 27 \mathrm{ac})$ of chromatin. ChIP'ed complexes were washed, sequentially treated with RNase $\left(30\right.$ minutes at $\left.37^{\circ} \mathrm{C}\right)$, proteinase $\mathrm{K}\left(30\right.$ minutes at $\left.55^{\circ} \mathrm{C}\right)$, de-crosslinking buffer $(1 \%$ SDS, $100 \mathrm{mM} \mathrm{NaHCO} 3$ (final concentration), 6-16 hours at $65^{\circ} \mathrm{C}$ ), and purified (\#28008, Qiagen). The concentration and size distribution of the immunoprecipitated DNA was measured using the Bioanalyzer High Sensitivity DNA kit (\#5067-4626, Agilent). Dana-Farber Cancer Institute Molecular Biology Core Facilities prepared libraries from 2 ng of DNA, using the ThruPLEX DNA-seq kit (\#R400427, Rubicon Genomics), according to the manufacturer's protocol; submitted the finished libraries to quality control analyses as described in the bulk RNA-seq Methods section; ChIP-seq libraries were uniquely indexed in equimolar ratios, and sequenced to a target depth of $40 \mathrm{M}$ reads on an Illumina NextSeq500 run, with single-end $75 \mathrm{bp}$ reads. mCRPC LuCaP PDXs: ChIP-sequencing for AR (N-20; \#sc-816, Santa Cruz Biotechnology), FOXA1 (\#ab23738, Abcam) and H3K27ac (\#C15410196, Diagenode), was performed at the DanaFarber Cancer Institute using the protocol described previously ${ }^{32,63}$.

LNCaP MYC model: Published gene expression data (GSE73995; 15) was downloaded and reanalyzed.

\section{Bioinformatics analyses - ChIP-seq}

Peak calling and data analysis: All samples were processed through the computational pipeline developed at the Dana-Farber Cancer Institute Center for Functional Cancer Epigenetics (CFCE) using primarily open source programs. Sequence tags were aligned with Burrows-Wheeler Aligner (BWA) to build mm9 or hg19 and uniquely mapped, non-redundant reads were retained ${ }^{64}$. These reads were used to generate binding sites with Model-Based Analysis of ChIP-seq 2 (MACS v2.1.1.20160309), with a q-value (FDR) threshold of $0.01^{65}$. We evaluated multiple quality control criteria based on alignment information and peak quality: (i) sequence quality score; (ii) uniquely mappable reads (reads that can only map to one location in the genome); (iii) uniquely mappable locations (locations that can only be mapped by at least one read); (iv) peak overlap with Velcro regions, a comprehensive set of locations - also called consensus signal artifact regions - in the genome that have anomalous, unstructured high signal or read counts in next-generation sequencing experiments independent of cell line and of type of experiment; (v) number of total peaks (the minimum required was 1,000); (vi) high-confidence peaks (the number of peaks that are tenfold enriched over background); (vii) percentage overlap with known DHS sites derived from the ENCODE Project (the minimum required to meet the threshold was $80 \%$ ); and (viii) peak conservation (a measure of sequence similarity across species based on the hypothesis that conserved sequences are more likely to be functional). Typically, if a sample fails one of these criteria, it will fail many (locations with low mappability will likely have low peak numbers, many of which will likely be in high-mappability regions, etc.). 
DNA binding motif analyses: Peaks from each group were used for motif analysis by the motif search findMotifsGenome.pl in HOMER (v3.0.0)31, with cutoff q-value $\leq 1 \mathrm{e}-10$.

630 Sample-sample correlation and differential peaks analysis: Sample-sample correlation and differential peaks analysis was performed by the CoBRA pipeline ${ }^{66}$. Peaks from all samples were merged to create a union set of sites for each transcription factor and histone mark. Read densities were calculated for each peak for each sample, which were used for comparison of cistromes across samples. Sample similarity was determined by hierarchical clustering using the Spearman correlation between samples. Tissue-specific peaks were identified by DEseq2 with adjusted $\mathrm{P} \leq$ 0.05 . Total number of reads in each sample was applied to size factor in DEseq2, which can normalize the sequencing depth between samples. bigwig files were calculated for each sample that reflected the coverage signal and sequencing depth using the Chilin pipeline ${ }^{67}$. The deepTools v.2.3.5 package computeMatrix further computed the average score for each of the samples. Finally, a profile heat map was created based on the scores at genomic positions within $2 \mathrm{~kb}$ upstream and downstream of the AR binding sites. All samples were ranked by the average score. ChIP-seq enrichment for transcription factors and histone marks at the loci of selected genes were visualized and plotted using karyoploteR (1.12.4) R package ${ }^{68}$. RNA Pol II analysis: RNA Pol II traveling ratio (TR) scores for each gene was calculated by comparing the ratio between RNA Pol II density in the promoter region and in the gene region ${ }^{41}$. The promoter region was defined as $-30 \mathrm{bp}$ to $+300 \mathrm{bp}$ relative to the transcriptional start site (TSS) and the gene body as the remaining length of the gene. We calculated the bins per million mapped reads (BPM) use bamCoverage and computeMatrix in deepTools v.2.3.5 for promoter and gene body regions. The TR difference between WT and MYC were calculated by TR value in WT minus TR value in MYC. Ranking plot of the WT MYC TR difference for all Pol II bound genes revealed a clear point in the distribution of travel ratio difference where the difference began increasing/decreasing rapidly. To geometrically define this point, we found the $\mathrm{x}$-axis point for which a line with a slope of 1 was tangent to the curve. We defined 246 genes above the increasing point to be pause release genes and 556 genes below the decreasing point to be the pause genes by MYC overexpression. DeepTools function plotProfile and plotHeatmap were used to create the Pol 658 II occupancy (the region $\pm 3 \mathrm{~kb}$ from the start and end of the gene) summary profiles and heatmaps. 659 Kolmogorov-Smirnov test is applied to the TR distribution difference between WT and MYC for 660 Hallmark Androgen_response genes.

\section{Epigenomics and transcriptomics integration}

All genes within the $100 \mathrm{~kb}$ of gained AR binding sites in MYC samples were selected, k-means clustering of 3 was applied. Cells were ordered by the pseudotime. GSEA analysis was done using the gene sets deposited in the GSEA website (https://www.gseamsigdb.org/gsea/msigdb/annotate.jsp). Binding and expression target analysis (BETA) was used to integrates ChIP-seq of transcription factors with differential gene expression data and infer the dysregulated genes ${ }^{39}$.

\section{Prostate cancer clinical datasets analyses}

669 The Cancer Genome Atlas (TCGA): RNA-seq readcount and clinical data from 488 samples with prostate cancer (PRAD) were downloaded from the cancer Genome Atlas (TCGA) database 671 (https://cancergenome.nih.gov/) using Bioconductor package TCGAbiolinks (2.14.1) ${ }^{69}$. To 672 calculate transcriptional signature scores, RNA-seq data was normalized to sequencing depth and 673 TPM transformed. Hallmark Androgen_response and Hallmark MYC_targets_V1 gene sets were 
674 downloaded from MSigDB ${ }^{70}$. The AR-A signature comprising nine canonical AR transcriptional 675 targets (KLK3, KLK2, FKBP 5, STEAP1, STEAP2, PPAP 2A, RAB3B, ACSL3, NKX3-1) was derived

676 from previous published work ${ }^{34}$. Transcriptional signature scores were computed for every patient 677 based on a non-parametric, rank-based method implemented in singscore (1.6.0) R package ${ }^{71}$.

678 TCGA patients were assigned to the low or high group according to the cut-off point estimated by 679 maximally selected rank statistic maxstat (0.7-25) R package of each signature ${ }^{72}$. Survival analysis 680 was conducted using survival (3.2-3) R package ${ }^{73}$, Kaplan-Meier were plotted using survminer 681 (0.4.8) R package ${ }^{74}$ and log-rank test was used to evaluate the overall statistical significance as 682 well as the comparison between groups. Benjamini-Hochberg was used to correct for multiple 683 testing.

684 Validation cohort: The META855 cohort containing 855 patients treated with radical 685 prostatectomy with available transcriptomic, clinicopathological, and outcomes data selected from 686 five published studies of the Decipher prostate genomic classifier test as previously described ${ }^{35}$. 687 Microarray expression levels were normalized using the SCAN algorithm (SCAN.UPC package) ${ }^{75}$. 688 The combination of the Hallmark Androgen_response / Hallmark MYC_targets_V1 and AR-A / 689 Hallmark MYC_targets_V1 signatures and their association with BCR and metastatic progression 690 was examined in the META855 cohort using the thresholds obtained from quantiles defined in the 691 TCGA dataset. Patients were divided in four groups and Kaplan-Meier analysis and log-rank test 692 were conducted to evaluate differences in biochemical recurrence and metastatic progression. The 693 prognostic association between the signatures and the clinicopathological factors was assessed 694 using Cox proportional hazard modeling.

695 Castration-resistant prostate cancer: Published gene expression data (GSE126078; ${ }^{37}$ ) was 696 downloaded and data analysis was performed using VIPER ${ }^{57}$.

\section{Data availability}

698 Data are available from the corresponding authors upon request. The murine (bulk RNA-seq, 699 scRNA-seq and ChIP-seq) and LuCaP PDXs (ChIP-seq) sequencing data reported in this paper 700 will be deposited on NCBI Gene Expression Omnibus (GEO). 
701

7021 Siegel, R. L., Miller, K. D. \& Jemal, A. Cancer statistics, 2019. CA Cancer J Clin 69, 7-34,

703

704

705

706

707

708

709

710

711

712

713

714

715

716

717

718

719

720

721

722

723

724

725

726

727

728

729

730

731

732

733

734

735

736

737

738

739

740

741

742

743

doi:10.3322/caac.21551 (2019).

2 White, J. W. II. The Present Position of the Surgery of the Hypertrophied Prostate. Ann Surg 18, 152-188 (1893).

3 White, J. W. I. The Results of Double Castration in Hypertrophy of the Prostate. Ann Surg 22, 1-80 (1895).

4 Huggins, C. \& Hodges, C. V. Studies on prostatic cancer - I The effect of castration, of estrogen and of androgen injection on serum phosphatases in metastatic carcinoma of the prostate. Cancer Res 1, 293-297 (1941).

5 Huggins, C., Stevens, R. E. \& Hodges, C. V. Studies on prostate cancer II The effects of castration on advanced carcinoma of the prostate gland. Arch Surg-Chicago 43, 209-223 (1941).

6 Watson, P. A., Arora, V. K. \& Sawyers, C. L. Emerging mechanisms of resistance to androgen receptor inhibitors in prostate cancer. Nat Rev Cancer 15, 701-711, doi:10.1038/nrc4016 (2015).

7 Labbé, D. P. \& Brown, M. Transcriptional Regulation in Prostate Cancer. Cold Spring Harb Perspect Med 8, doi:10.1101/cshperspect.a030437 (2018).

8 Baca, S. C. et al. Punctuated evolution of prostate cancer genomes. Cell 153, 666-677, doi:10.1016/j.cell.2013.03.021 (2013).

9 Cancer Genome Atlas Research, N. The Molecular Taxonomy of Primary Prostate Cancer. Cell 163, 1011-1025, doi:10.1016/j.cell.2015.10.025 (2015).

10 Gurel, B. et al. Nuclear MYC protein overexpression is an early alteration in human prostate carcinogenesis. Mod Pathol 21, 1156-1167, doi:10.1038/modpathol.2008.111 (2008).

11 Matejcic, M. et al. Germline variation at $8 \mathrm{q} 24$ and prostate cancer risk in men of European ancestry. Nat Commun 9, 4616, doi:10.1038/s41467-018-06863-1 (2018).

12 Pomerantz, M. M. et al. The 8q24 cancer risk variant rs6983267 shows long-range interaction with MYC in colorectal cancer. Nat Genet 41, 882-884, doi:10.1038/ng.403 (2009).

13 Wasserman, N. F., Aneas, I. \& Nobrega, M. A. An 8q24 gene desert variant associated with prostate cancer risk confers differential in vivo activity to a MYC enhancer. Genome Res 20, 1191-1197, doi:10.1101/gr.105361.110 (2010).

14 Ellwood-Yen, K. et al. Myc-driven murine prostate cancer shares molecular features with human prostate tumors. Cancer Cell 4, 223-238 (2003).

15 Barfeld, S. J. et al. c-Myc Antagonises the Transcriptional Activity of the Androgen Receptor in Prostate Cancer Affecting Key Gene Networks. EBioMedicine 18, 83-93, doi:10.1016/j.ebiom.2017.04.006 (2017).

16 Bostwick, D. G., Liu, L., Brawer, M. K. \& Qian, J. High-grade prostatic intraepithelial neoplasia. Rev Urol 6, 171-179 (2004).

17 Labbé, D. P. et al. High-fat diet fuels prostate cancer progression by rewiring the metabolome and amplifying the MYC program. Nat Commun 10, 4358, doi:10.1038/s41467-019-12298-z (2019). 
Crowell, P. D. et al. Expansion of Luminal Progenitor Cells in the Aging Mouse and Human Prostate. Cell Rep 28, 1499-1510 e1496, doi:10.1016/j.celrep.2019.07.007 (2019).

Dahlman, A. et al. Effect of androgen deprivation therapy on the expression of prostate cancer biomarkers MSMB and MSMB-binding protein CRISP3. Prostate Cancer Prostatic Dis 13, 369-375, doi:10.1038/pcan.2010.25 (2010).

Zhang, J., Thomas, T. Z., Kasper, S. \& Matusik, R. J. A small composite probasin promoter confers high levels of prostate-specific gene expression through regulation by androgens and glucocorticoids in vitro and in vivo. Endocrinology 141, 4698-4710, doi:10.1210/endo.141.12.7837 (2000).

753

21 Dang, C. V. MYC, metabolism, cell growth, and tumorigenesis. Cold Spring Harb Perspect Med 3, doi:10.1101/cshperspect.a014217 (2013).

755

Subramanian, A. et al. Gene set enrichment analysis: a knowledge-based approach for interpreting genome-wide expression profiles. Proc Natl Acad Sci U S A 102, 15545-15550, doi:10.1073/pnas.0506580102 (2005).

Azizi, E. et al. Single-Cell Map of Diverse Immune Phenotypes in the Breast Tumor Microenvironment. Cell 174, 1293-1308 e1236, doi:10.1016/j.cell.2018.05.060 (2018).

Mezzetti, G., Loor, R. \& Liao, S. Androgen-sensitive spermine-binding protein of rat ventral prostate. Purification of the protein and characterization of the hormonal effect. Biochem J 184, 431-440, doi:10.1042/bj1840431 (1979).

Pihlajamaa, P. et al. Tissue-specific pioneer factors associate with androgen receptor cistromes and transcription programs. Embo J 33, 312-326, doi:10.1002/embj.201385895 (2014).

Gross, M. et al. Beta-2-microglobulin is an androgen-regulated secreted protein elevated in serum of patients with advanced prostate cancer. Clin Cancer Res 13, 1979-1986, doi:10.1158/1078-0432.CCR-06-1156 (2007). mouse (Mus musculus musculus) in relation to chemical signalling. PeerJ 5, e3541, doi:10.7717/peerj.3541 (2017). Wubah, J. A. et al. Ventral prostate predominant I, a novel mouse gene expressed exclusively in the prostate. Prostate 51, 21-29, doi:10.1002/pros.10060 (2002).

29 Rasanen, K., Itkonen, O., Koistinen, H. \& Stenman, U. H. Emerging Roles of SPINK1 in Cancer. Clin Chem 62, 449-457, doi:10.1373/clinchem.2015.241513 (2016).

30 Wang, R. et al. Preclinical Study using Malat1 Small Interfering RNA or Androgen Receptor Splicing Variant 7 Degradation Enhancer ASC-J9((R)) to Suppress Enzalutamide-resistant Prostate Cancer Progression. Eur Urol 72, 835-844, doi:10.1016/j.eururo.2017.04.005 (2017).

31 van Riggelen, J., Yetil, A. \& Felsher, D. W. MYC as a regulator of ribosome biogenesis and protein synthesis. Nat Rev Cancer 10, 301-309, doi:10.1038/nrc2819 (2010).

783

784

Pomerantz, M. M. et al. The androgen receptor cistrome is extensively reprogrammed in human prostate tumorigenesis. Nat Genet 47, 1346-1351, doi:10.1038/ng.3419 (2015). Nature 473, 43-49, doi:10.1038/nature09906 (2011). 
34 Spratt, D. E. et al. Transcriptomic Heterogeneity of Androgen Receptor Activity Defines a de novo low AR-Active Subclass in Treatment Naive Primary Prostate Cancer. Clin Cancer Res 25, 6721-6730, doi:10.1158/1078-0432.CCR-19-1587 (2019).

35 Spratt, D. E. et al. Individual Patient-Level Meta-Analysis of the Performance of the Decipher Genomic Classifier in High-Risk Men After Prostatectomy to Predict Development of Metastatic Disease. J Clin Oncol 35, 1991-1998, doi:10.1200/JCO.2016.70.2811 (2017).

36 Taylor, B. S. et al. Integrative genomic profiling of human prostate cancer. Cancer Cell 18, 11-22, doi:10.1016/j.ccr.2010.05.026 (2010).

37 Labrecque, M. P. et al. Molecular profiling stratifies diverse phenotypes of treatmentrefractory metastatic castration-resistant prostate cancer. J Clin Invest 129, 4492-4505, doi:10.1172/JCI128212 (2019).

38 Nguyen, H. M. et al. LuCaP Prostate Cancer Patient-Derived Xenografts Reflect the Molecular Heterogeneity of Advanced Disease an--d Serve as Models for Evaluating Cancer Therapeutics. Prostate 77, 654-671, doi:10.1002/pros.23313 (2017).

39 Wang, S. et al. Target analysis by integration of transcriptome and ChIP-seq data with BETA. Nat Protoc 8, 2502-2515, doi:10.1038/nprot.2013.150 (2013).

40 Pomerantz, M. M. et al. Analysis of the 10q11 cancer risk locus implicates MSMB and NCOA4 in human prostate tumorigenesis. PLoS Genet 6, e1001204, doi:10.1371/journal.pgen.1001204 (2010).

41 Rahl, P. B. et al. c-Myc regulates transcriptional pause release. Cell 141, 432-445, doi:10.1016/j.cell.2010.03.030 (2010).

42 Chen, F. X., Smith, E. R. \& Shilatifard, A. Born to run: control of transcription elongation by RNA polymerase II. Nat Rev Mol Cell Biol 19, 464-478, doi:10.1038/s41580-018-0010-5 (2018).

$43 \mathrm{Ni}, \mathrm{M}$. et al. Amplitude modulation of androgen signaling by c-MYC. Genes Dev 27, 734748, doi:10.1101/gad.209569.112 (2013).

44 Karthaus, W. R. et al. Regenerative potential of prostate luminal cells revealed by singlecell analysis. Science 368, 497-505, doi:10.1126/science.aay0267 (2020).

45 Joseph, D. B. et al. Urethral luminal epithelia are castration-insensitive cells of the proximal prostate. Prostate, doi:10.1002/pros.24020 (2020).

46 Zhang, J. et al. Characterization of cis elements of the probasin promoter necessary for prostate-specific gene expression. Prostate 70, 934-951, doi:10.1002/pros.21128 (2010).

47 Harris, A. W. et al. The E mu-myc transgenic mouse. A model for high-incidence spontaneous lymphoma and leukemia of early B cells. J Exp Med 167, 353-371, doi:10.1084/jem.167.2.353 (1988).

48 Kumar, A. et al. Substantial interindividual and limited intraindividual genomic diversity among tumors from men with metastatic prostate cancer. Nat Med 22, 369-378, doi:10.1038/nm.4053 (2016).

49 Antonarakis, E. S. et al. AR-V7 and resistance to enzalutamide and abiraterone in prostate cancer. N Engl J Med 371, 1028-1038, doi:10.1056/NEJMoa1315815 (2014).

50 Joseph, J. D. et al. A clinically relevant androgen receptor mutation confers resistance to second-generation antiandrogens enzalutamide and ARN-509. Cancer Discov 3, 1020 1029, doi:10.1158/2159-8290.CD-13-0226 (2013). 
$83051 \quad$ Arriaga, J. M. et al. A MYC and RAS co-activation signature in localized prostate cancer drives bone metastasis and castration resistance. Nature Cancer 1, 1082-1096, doi:10.1038/s43018-020-00125-0 (2020).

833

52 Bai, S. et al. A positive role of c-Myc in regulating androgen receptor and its splice variants in prostate cancer. Oncogene 38, 4977-4989, doi:10.1038/s41388-019-0768-8 (2019).

Dardenne, E. et al. N-Myc Induces an EZH2-Mediated Transcriptional Program Driving Neuroendocrine Prostate Cancer. Cancer Cell 30, 563-577, doi:10.1016/j.ccell.2016.09.005 (2016).

83854 Malynn, B. A. et al. N-myc can functionally replace c-myc in murine development, cellular growth, and differentiation. Genes Dev 14, 1390-1399 (2000).

55 Schmidt, S. F., Larsen, B. D., Loft, A. \& Mandrup, S. Cofactor squelching: Artifact or fact? Bioessays 38, 618-626, doi:10.1002/bies.201600034 (2016).

Han, H. et al. Small-Molecule MYC Inhibitors Suppress Tumor Growth and Enhance Immunotherapy. Cancer Cell 36, 483-497 e415, doi:10.1016/j.ccell.2019.10.001 (2019).

Cornwell, M. et al. VIPER: Visualization Pipeline for RNA-seq, a Snakemake workflow for efficient and complete RNA-seq analysis. BMC Bioinformatics 19, 135, doi:10.1186/s12859-018-2139-9 (2018).

Dobin, A. et al. STAR: ultrafast universal RNA-seq aligner. Bioinformatics 29, 15-21, doi:10.1093/bioinformatics/bts635 (2013).

Trapnell, C. et al. Transcript assembly and quantification by RNA-Seq reveals unannotated transcripts and isoform switching during cell differentiation. Nat Biotechnol 28, 511-515, doi:10.1038/nbt.1621 (2010).

862

863

Love, M. I., Huber, W. \& Anders, S. Moderated estimation of fold change and dispersion for RNA-seq data with DESeq2. Genome Biol 15, 550, doi:10.1186/s13059-014-0550-8 (2014). transcriptomic data across different conditions, technologies, and species. Nat Biotechnol 36, 411-420, doi:10.1038/nbt.4096 (2018).

$62 \mathrm{Gu}$, Z., Eils, R. \& Schlesner, M. Complex heatmaps reveal patterns and correlations in multidimensional genomic data. Bioinformatics 32, 2847-2849, doi:10.1093/bioinformatics/btw313 (2016). during metastatic progression. Nat Genet 52, 790-799, doi:10.1038/s41588-020-0664-8 (2020). alignment of short DNA sequences to the human genome. Genome Biol 10, R25, doi:10.1186/gb-2009-10-3-r25 (2009).

5 Zhang, Y. et al. Model-based analysis of ChIP-Seq (MACS). Genome biology 9, R137, doi:10.1186/gb-2008-9-9-r137 (2008).

66 Qiu, X. et al. CoBRA: Containerized Bioinformatics workflow for Reproducible ChIP/ATACseq Analysis - from differential peak calling to pathway analysis. bioRxiv, 2020.2011.2006.367409, doi:10.1101/2020.11.06.367409 (2020).

67 Qin, Q. et al. ChiLin: a comprehensive ChIP-seq and DNase-seq quality control and analysis pipeline. BMC Bioinformatics 17, 404, doi:10.1186/s12859-016-1274-4 (2016). 
87468 Gel, B. \& Serra, E. karyoploteR: an R/Bioconductor package to plot customizable genomes

875 displaying arbitrary data. Bioinformatics 33, 3088-3090,

876 doi:10.1093/bioinformatics/btx346 (2017).

87769 Colaprico, A. et al. TCGAbiolinks: an R/Bioconductor package for integrative analysis of

878 TCGA data. Nucleic Acids Res 44, e71, doi:10.1093/nar/gkv1507 (2016).

87970 Liberzon, A. et al. The Molecular Signatures Database (MSigDB) hallmark gene set $880 \quad$ collection. Cell Syst 1, 417-425, doi:10.1016/j.cels.2015.12.004 (2015).

88171 Foroutan, M. et al. Single sample scoring of molecular phenotypes. BMC Bioinformatics

88219,404 , doi:10.1186/s12859-018-2435-4 (2018).

88372 Hothorn, T. maxstat: Maximally Selected Rank Statistics. R Package Version 07-25. Available online: https://cran.r-project.org/web/packages/maxstat/. (2017).

73 Therneau, T. A., Lumley, T., Elizabeth, A. \& Cynthia, C. survival: Survival Analysis. R Package Version 3.2-3. Available online: https://cran.r-project.org/web/packages/survival/. (2020).

889

74 Kassambara, A., Kosinski, M., Biecek, P. \& Fabian, S. survminer: Drawing Survival Curves using 'ggplot2'. R package version 0.4.8. Available online: https://cran.rproject.org/web/packages/survminer/. (2020).

892

Piccolo, S. R. et al. A single-sample microarray

893 personalized-medicine workflows.

Genomics

100,

facilitate doi:10.1016/j.ygeno.2012.08.003 (2012). 
bioRxiv preprint doi: https://doi.org/10.1101/2021.04.23.441016; this version posted April 24, 2021. The copyright holder for this preprint (which was not certified by peer review) is the author/funder, who has granted bioRxiv a license to display the preprint in perpetuity. It is made available under aCC-BY 4.0 International license.

\section{FIGURES}

A

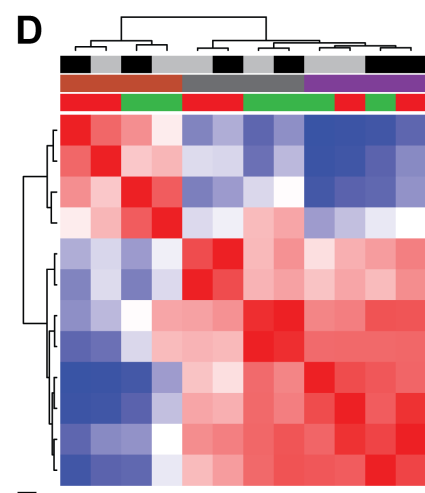

D

$\mathbf{F}$

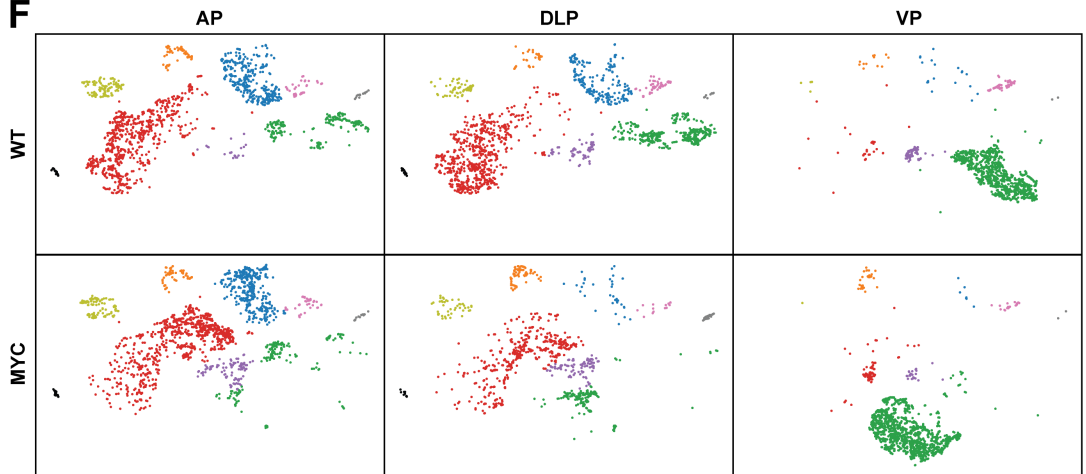

G

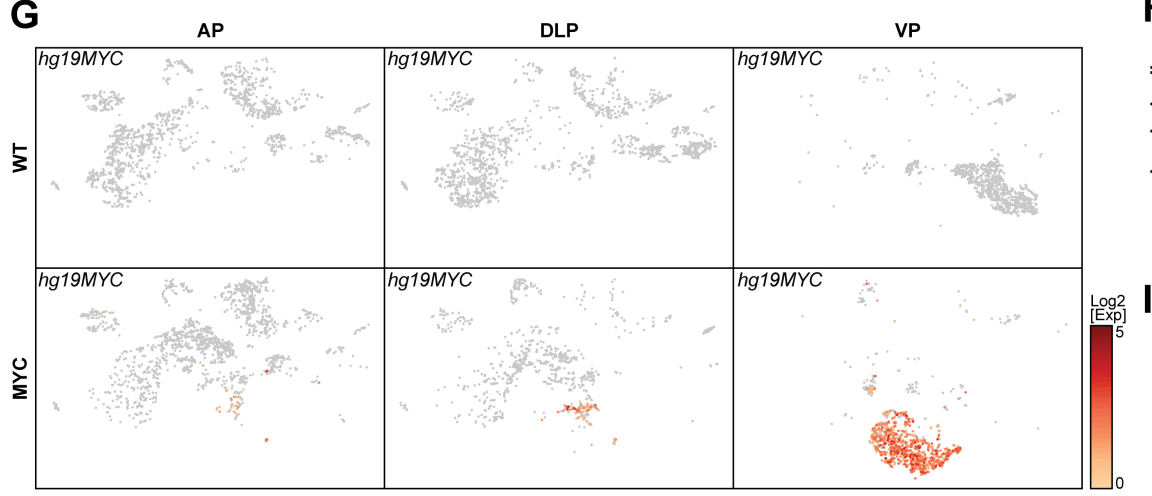

Figure 1
B

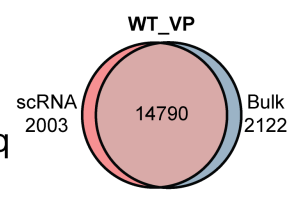

VP $\longrightarrow$ bulk RNA-seq

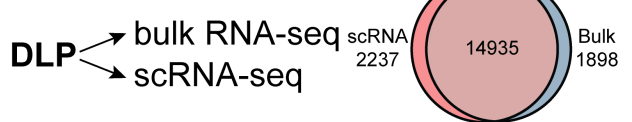
MYC_DLP scRNA-seq
Sequencing Genotype MYC_VP scRNA-seq $\quad$ Sequencing MYC_VP bulk RNA-seq WT_VP scRNA-seq WT VP bulk RNA-seq MYC DLP bulk RNA-seq WT_DLP scRNA-seq WT_DLP bulk RNA-seq WT_AP bulk RNA-seq MYC_AP bulk RNA-seq MYC AP scRNA-seq WT_AP scRNA-seq - scRNA-seq

Lobe

AP

DLP
VP

Genotype

MYC Correlation -1
-0.9

0.8

-0.7
-0.6
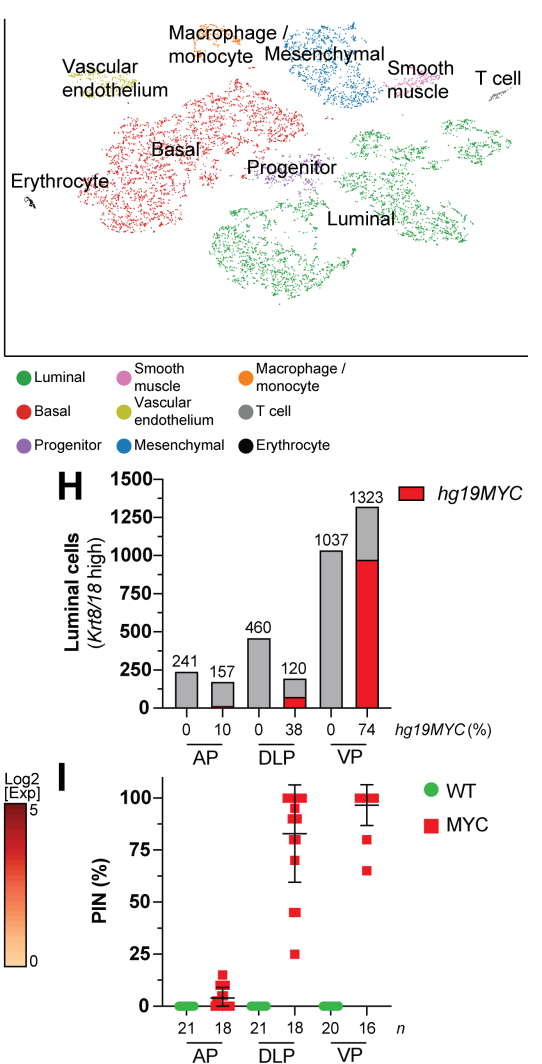
896 Figure 1: MYC induces a profound transcriptional reprogramming in murine prostate lobes.

897 (A) Graphical summary of the experimental design. (B, C) Transcriptional profiling of WT and 898 MYC-transformed VP reveal high concordance for the total number of genes detected (B) and their 899 expression levels (C) between bulk and single-cell RNA-seq. (D, E) Sample-sample correlation 900 (D) and principal component analysis (E) between bulk and matched single-cell transcriptome 901 identifies distinct transcriptional profiles across murine prostate lobes. (F) Single-cell census of 902 WT and MYC-transformed AP, DLP and VP. tSNE of scRNA-seq profiles colored using known 903 markers identified nine major subpopulations across prostate lobes. (G-I) The human MYC 904 transgene (hg19MYC) expression is largely restricted to the luminal compartment (G) and 905 predominantly expressed in the VP $(\mathbf{H})$, in accordance with the penetrance of prostatic 906 intraepithelial neoplasia (I, PIN; mean \pm SD; $\left.{ }^{17}\right)$. WT: wild-type; VP: ventral prostate; DLP: 907 dorsolateral prostate; AP: anterior prostate. 


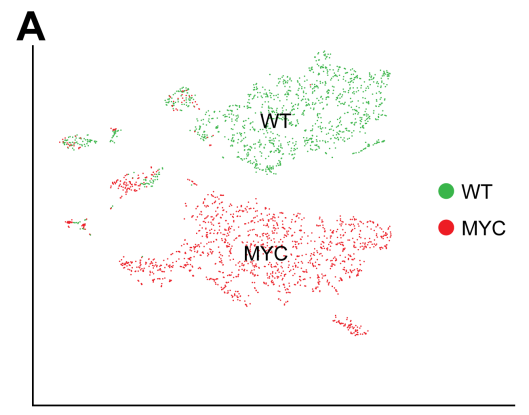

C

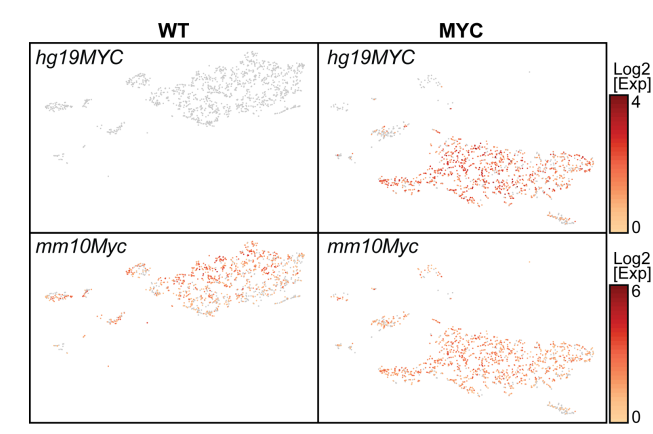

908

909

910

911

912

913

914 hg19MYC expression in luminal cells (D).
B

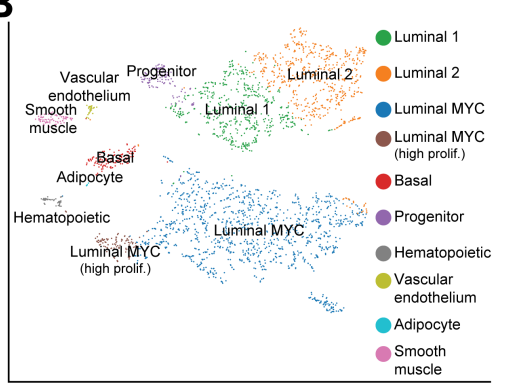

D $\left.1.5\right|^{P=2.100 .65: F R=60.010 .61}$

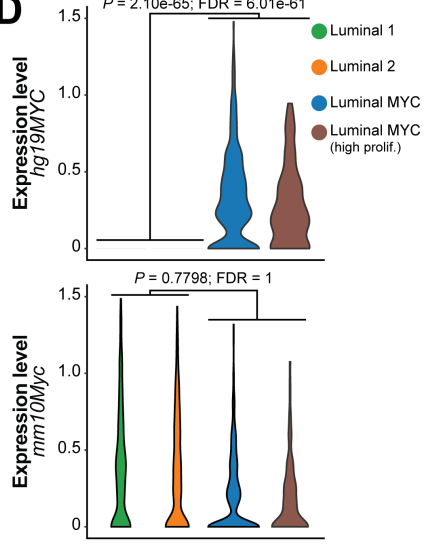

Figure 2: Single-cell transcriptome reveals distinct luminal cell subpopulations. (A, B) Single cell census of the WT and MYC-transformed VP (A) followed by unsupervised clustering revealed four luminal subsets (B). (C, D) Human MYC transcript ( $h g 19 M Y C)$ is only observed in MYCtransformed VP and mostly restricted to the luminal subsets while murine $M y c$ transcript $(m m 10 M y c)$ is expressed across cellular populations and genotypes $(\mathbf{C})$ and is not correlated with 


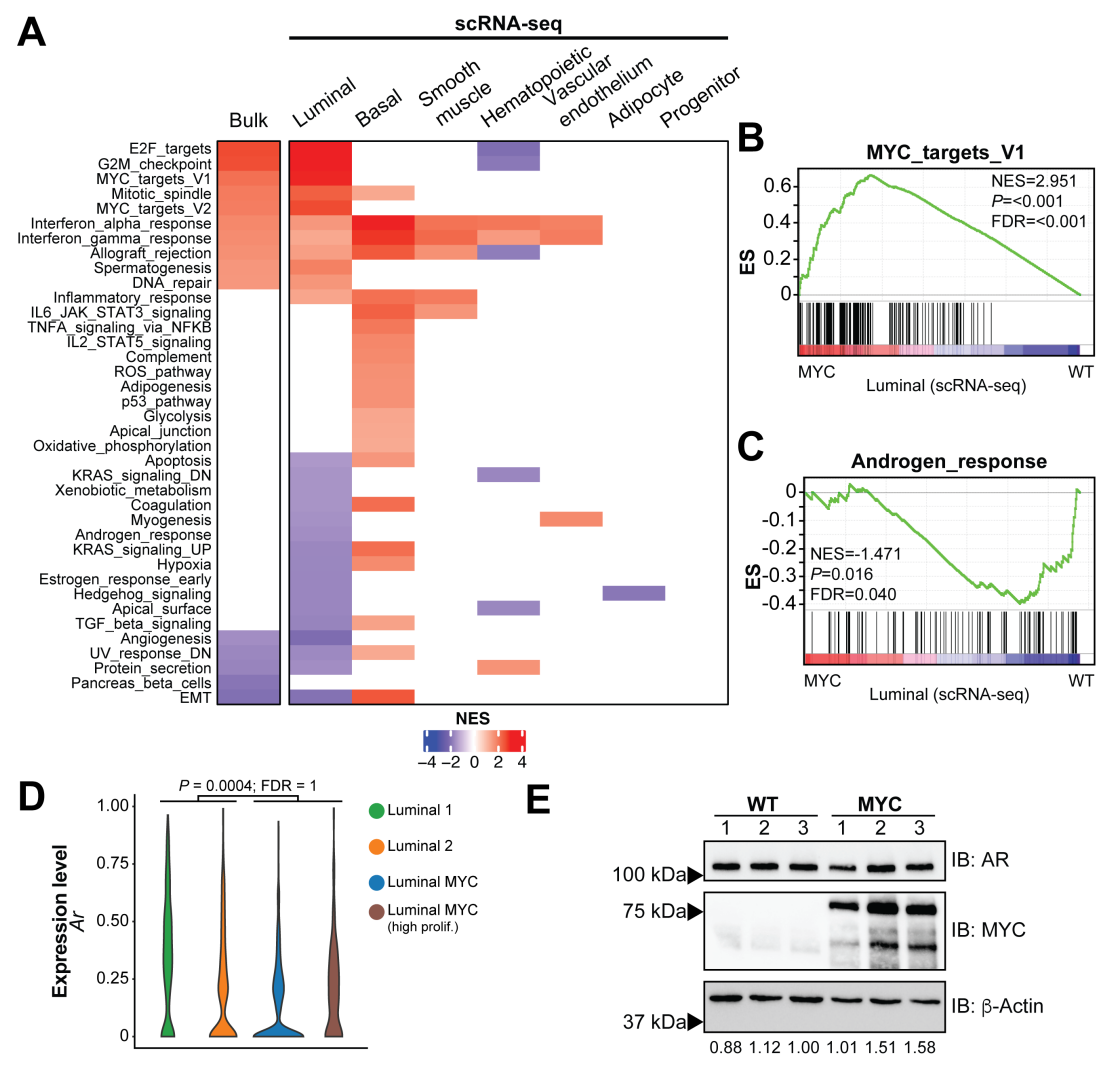

$\mathbf{F}$

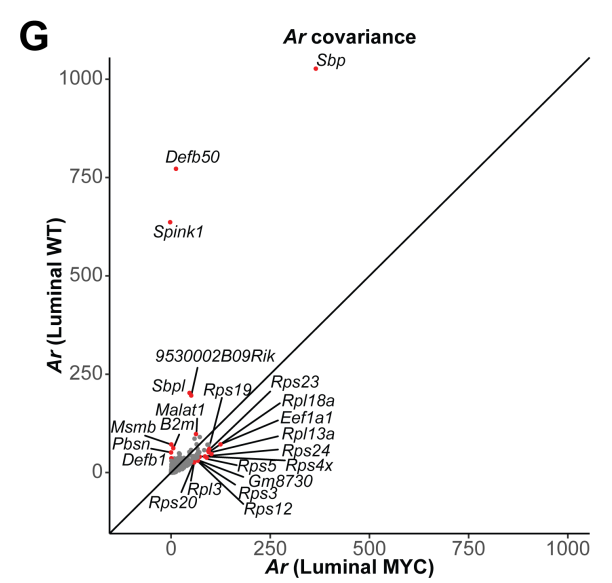

Figure 3: MYC-driven luminal cells transformation dampens the AR transcriptional program. (A) Gene Set Enrichment Analysis (GSEA, Hallmark, $\mathrm{P}<0.05$ and FDR $<0.1$ ) revealed that the bulk RNA-seq transcriptional program associated with MYC overexpression is mostly driven by the luminal subset (matched scRNA-seq). (B, C) MYC overexpression is associated with an enriched MYC transcriptional program (B) and a depleted AR response (C) in the luminal subsets. (D, E) MYC overexpression does not alter $\mathrm{AR}$ transcript expression in the luminal compartment (D) and protein levels in the VP (E; numbers at the bottom represent AR levels relative to $\beta$-Actin). (F) Schematic representation of covariance analysis to determine coexpression (i.e. positive covariance) or mutually exclusive expression (i.e. negative covariance) between two genes at a single cell level. (G) Covariance analysis in the luminal subset reveals a shift from canonical AR target genes in the transcripts co-expressed with $A r$ upon MYC overexpression. 
bioRxiv preprint doi: https://doi.org/10.1101/2021.04.23.441016; this version posted April 24, 2021. The copyright holder for this preprint (which was not certified by peer review) is the author/funder, who has granted bioRxiv a license to display the preprint in perpetuity. It is made available under aCC-BY 4.0 International license.

A

AR ChIP-seq top binding motif ARE(NR)/LNCAP-AR-ChIP-seq(GSE27824)/Homer

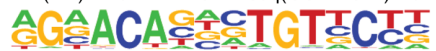

WT_1: $P=1 \mathrm{e}-1304$

WT-2: $P=1 \mathrm{e}-1014$

MYC_1: $P=1 \mathrm{e}-991$
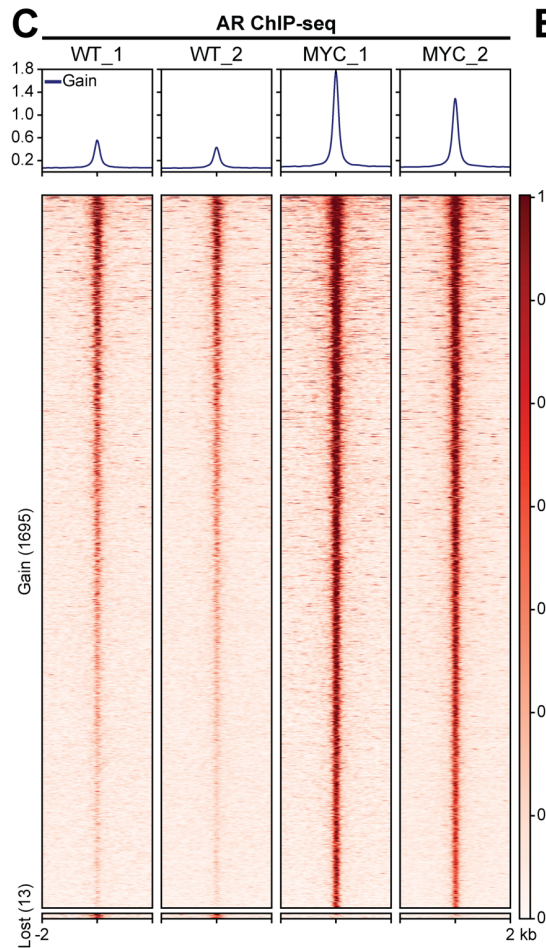

G

Figure 4
B
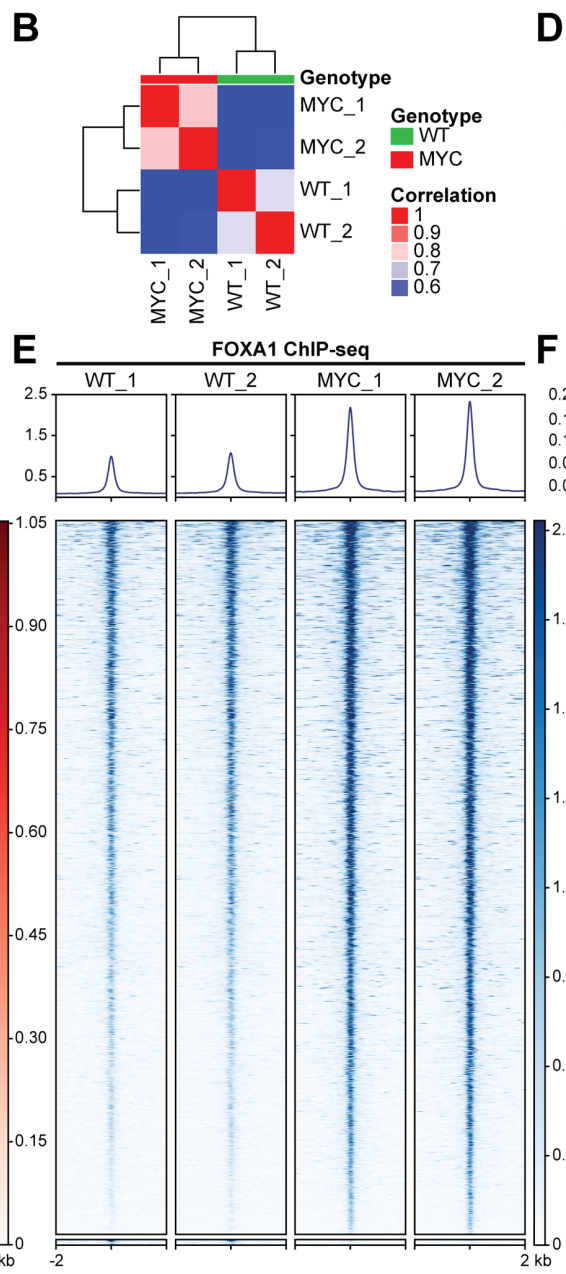

H Luminal subpopulation

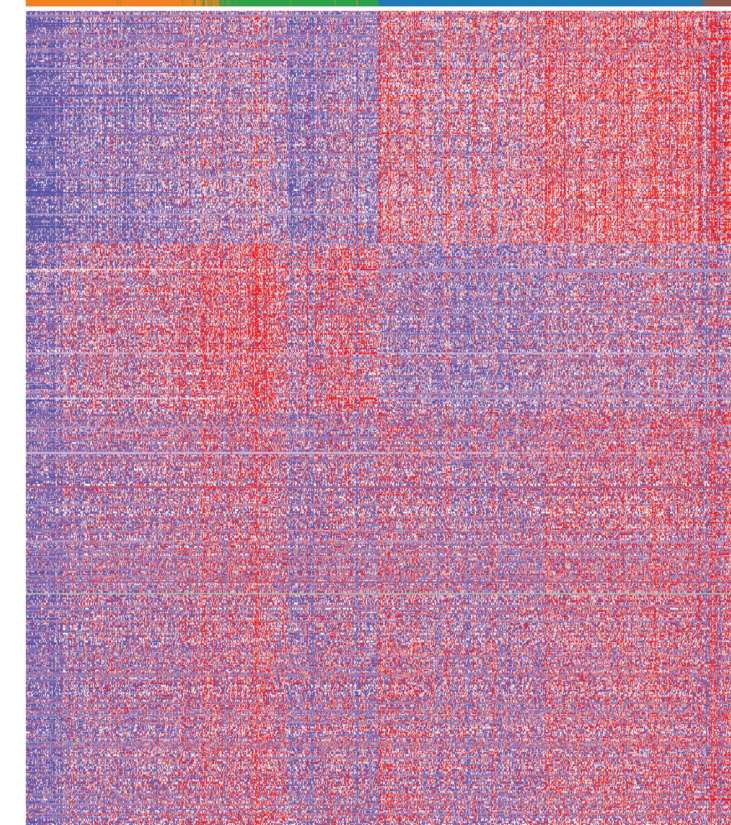

D

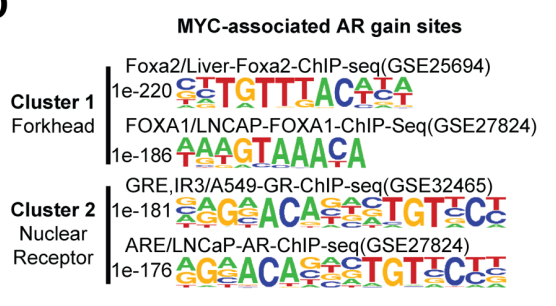

$F$

$\frac{\text { H3K27ac ChIP-seq }}{\text { WT_1 }}$
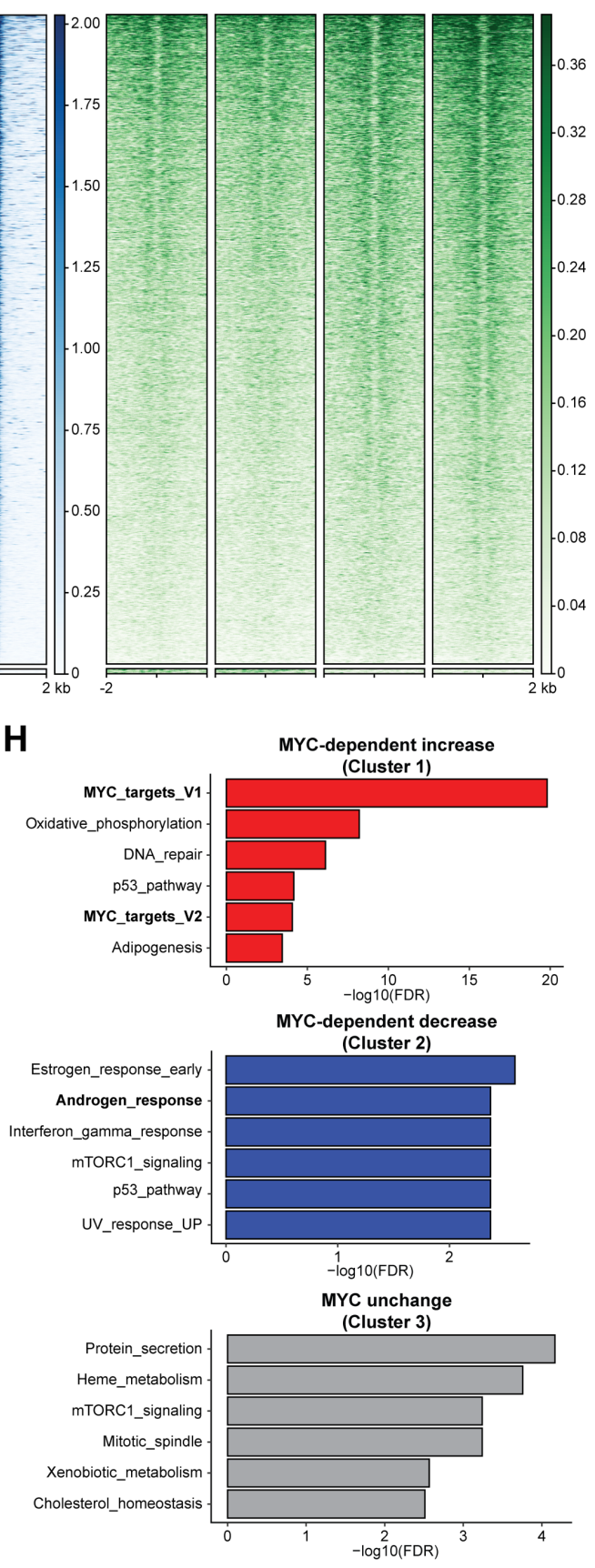
929 Figure 4: MYC overexpression alters the AR cistrome. (A) AR ChIP-seq identifies an androgen 930 response element (ARE) as the top AR binding motif in WT and MYC-transformed VP. (B) 931 Unsupervised pairwise correlation of the murine AR cistrome from all specimens. (C) MYC 932 overexpression expands the AR cistrome as demonstrated by the heatmaps indicating AR binding 933 intensity across $4 \mathrm{~kb}$ intervals. (D) Motif analysis of MYC-associated AR gained sites reveal 934 forkhead response element (FHRE) and androgen response element (ARE). (E, F) AR gained sites 935 are characterized by increased FOXA1 binding $(\mathbf{E})$ and H3K27ac mark $(\mathbf{F})$ in MYC-transformed 936 VP. (G) Integration of the 1,695 AR bindings sites gained in MYC tumors with luminal single cell 937 transcriptome grouped by k-means clustering $(n=3)$. (H) GSEA analysis (Hallmark) revealed an 938 enforced MYC transcriptional program (Cluster 1) and a diminished androgen response (Cluster 939 2) associated to MYC-dependent AR gained binding sites. 
A

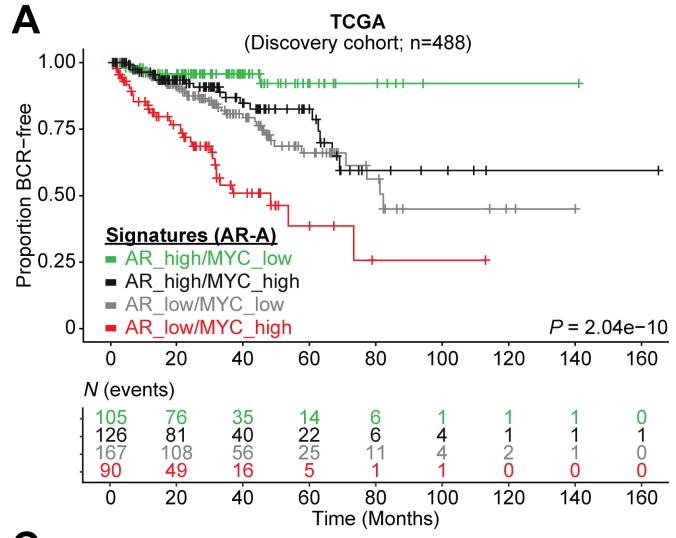

B Signatures AR_low/ AR_low/ AR_high/ AR_high/

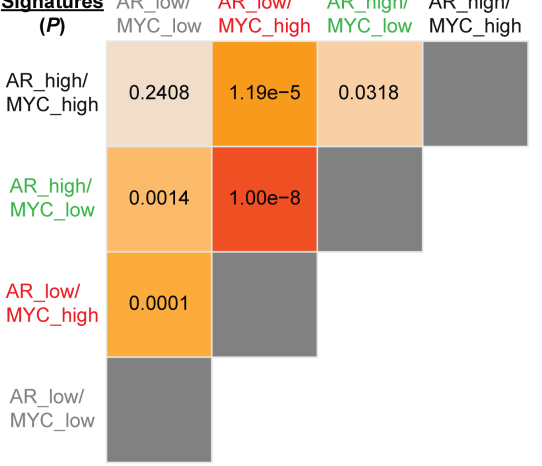

C
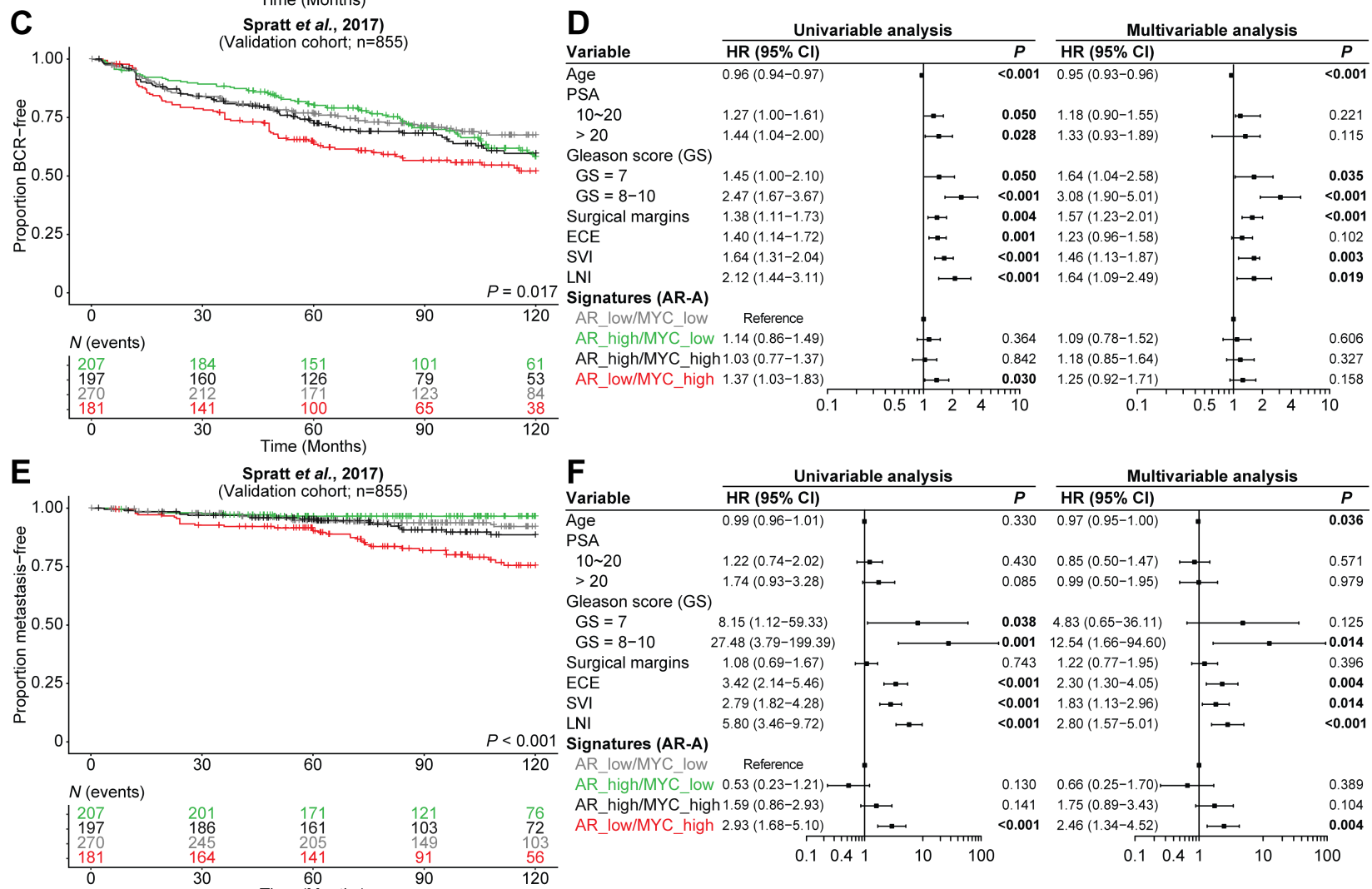

Figure 5: Divergent MYC and AR transcriptional programs dictate disease progression. (A,

B) Kaplan-Meier curves (A) and log-rank tests (B) reveal that patients bearing a primary tumor characterized by low AR-activity (AR-A) and concurrent high MYC transcriptional signature (Hallmark) have a shorter time to biochemical recurrence (BCR) within the discovery cohort (TCGA). (C, D) Kaplan-Meier curves (C), univariable and multivariable analysis (D) confirms that tumors with concurrent low AR-A and high MYC transcriptional signatures develop BCR after radical prostatectomy more rapidly than low AR-A tumors without an active MYC transcriptional program in the validation cohort (Spratt et al., 2017). (E, F) Kaplan-Meier curves (E), univariable and multivariable analyses (F) reveal that tumors with concurrent low AR-A and high MYC transcriptional signatures are more likely to develop a metastatic disease. PSA: prostate-specific antigen; HR: hazard ratio; GS: Gleason score; ECE: extracapsular extension; SVI: seminal vesicles invasion; LNI: lymph node involvement. 
A

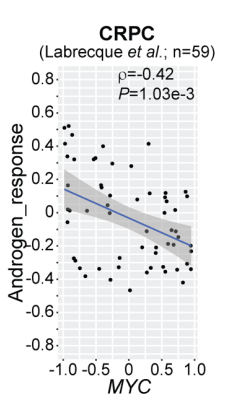

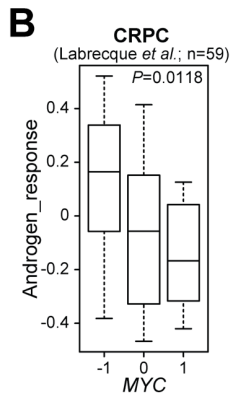

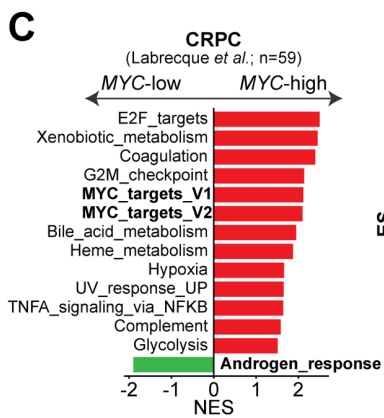

F

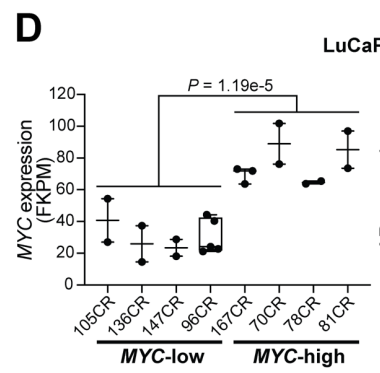

E

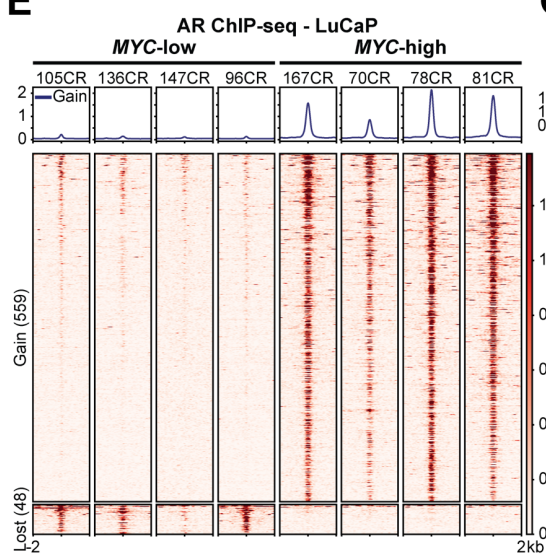

$P=0.0027$

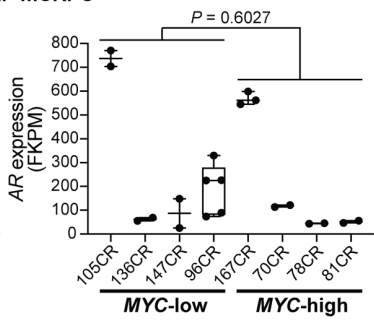

G

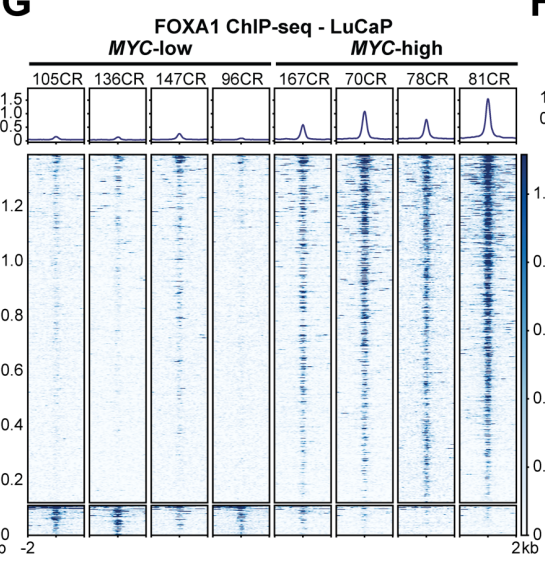

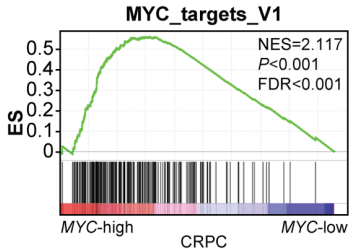

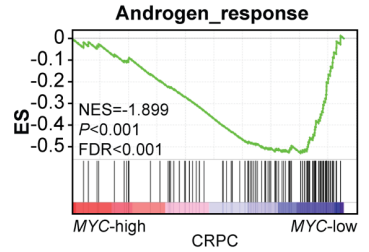

I
MYC-high AR gain sites

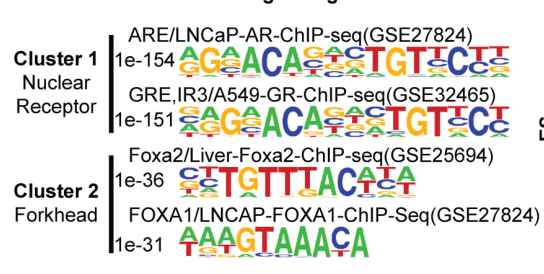

H

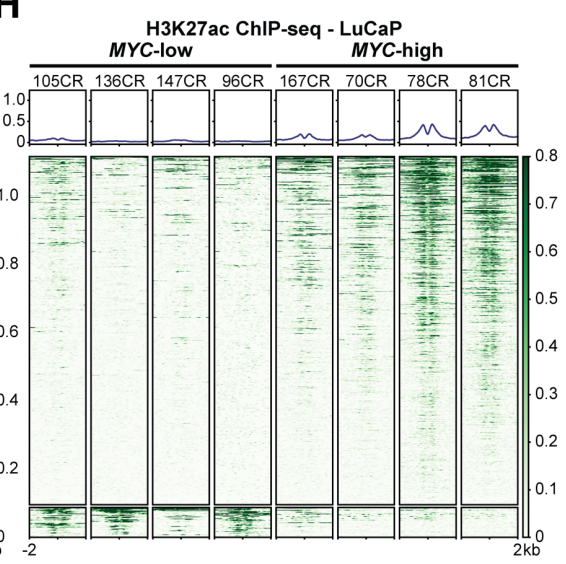

954 Figure 6: High MYC expression is associated with a dampened AR transcriptional program and an expanded $A R$ cistrome in castration-resistant tumors. (A, B) AR activity is inversely correlated with $M Y C$ expression in CRPC clinical samples (A) and significantly lower in $M Y C$ high tumors (B; median \pm 1.5 interquartile range). (C) Gene Set Enrichment Analysis (GSEA, Hallmark, $\mathrm{P}<0.05$ and FDR $<0.1$ ) revealed an enriched MYC transcriptional program and a depleted AR response in $M Y C$-high CRPC. (D, E) $M Y C$-high mCRPC LuCaP patient-derived xenografts (PDXs) have similar levels of $A R$ (D; median \pm min to max) but are associated with an expanded AR cistrome as demonstrated by the increased binding intensity across $4 \mathrm{~kb}$ intervals at AR gained sites (E). (F) Motif analysis of $M Y C$-associated AR gained sites reveal ARE and FHRE. (G, H) AR gained sites are characterized by increased FOXA1 binding $(\mathbf{G})$ and H3K27ac mark $(\mathbf{H})$ in a diminished androgen response. 
bioRxiv preprint doi: https://doi.org/10.1101/2021.04.23.441016; this version posted April 24, 2021. The copyright holder for this preprint (which was not certified by peer review) is the author/funder, who has granted bioRxiv a license to display the preprint in perpetuity. It is made available under aCC-BY 4.0 International license.

A BETA of AR peaks vs. MYC dysregulated genes

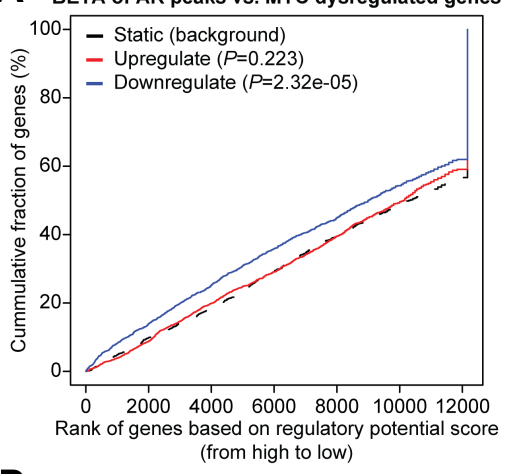

B

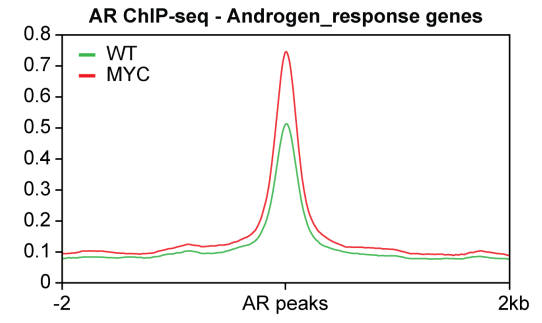

C

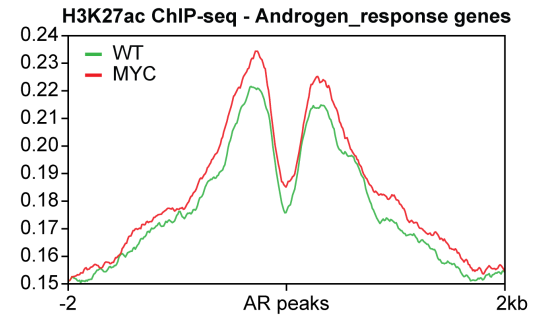

D (from high to low)

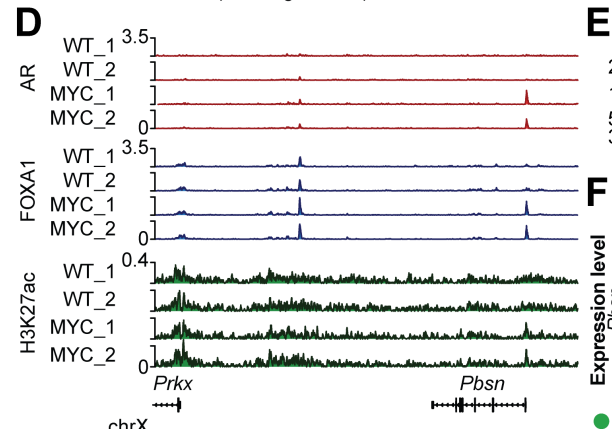

E $\quad \frac{\text { WT }}{123} \frac{\text { MYC }}{123}$

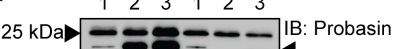
$10 \mathrm{kDa}$ $30 \mathrm{kDa}$ F $\quad \begin{aligned} & P=5.88 \mathrm{e}-95 \\ & \mathrm{FDR}=1.02 \mathrm{e}-90\end{aligned}$ G $\begin{aligned} & P=3.46 e-63 \\ & F D R=5.99 \mathrm{e}-59\end{aligned}$

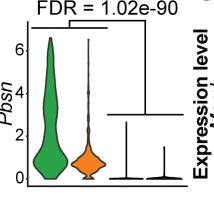

H

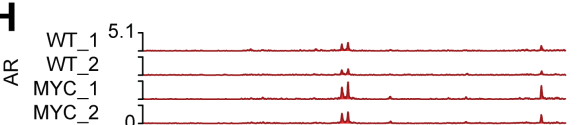

MYC 2

WT ${ }^{5.1}$

这 WT_2

MYC_1

MYC_2

MT 10.85

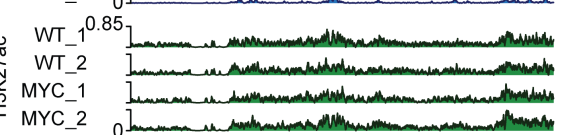

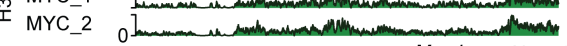

Msmb Ncoa4

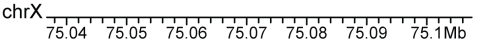
Luminal 1 Luminal 2 Luminal MYC $\stackrel{\text { Luminal MYC }}{\text { (high prolif.) }}$

chr14

$\begin{array}{llllll}32.93 & 32.94 & 32.95 & 32.96 & 32.97 & 32.98 \mathrm{M}\end{array}$

I

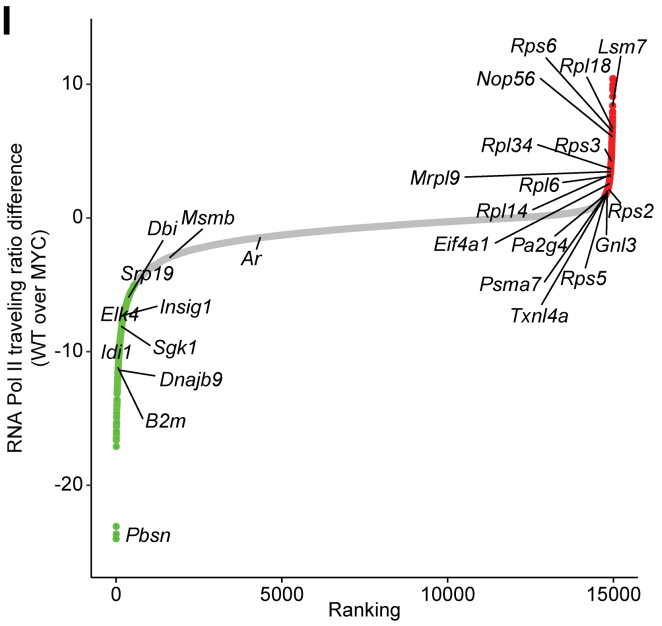

J RNA Pol II ChIP-seq - Pause release in MYC
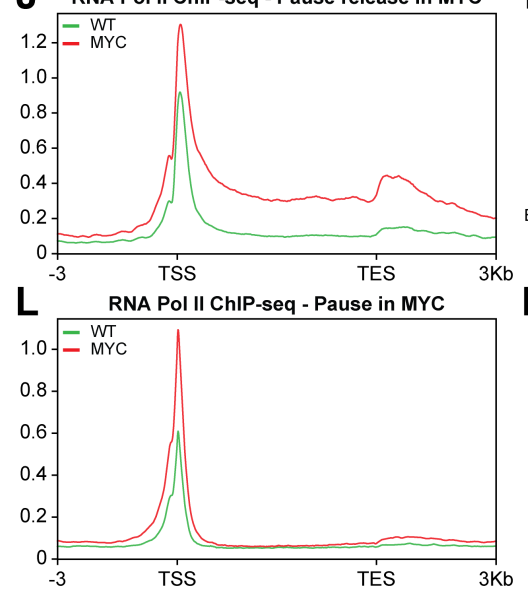

$\mathbf{M}$

0

$\mathbf{P}$

$\mathbf{Q}_{\text {s }}$

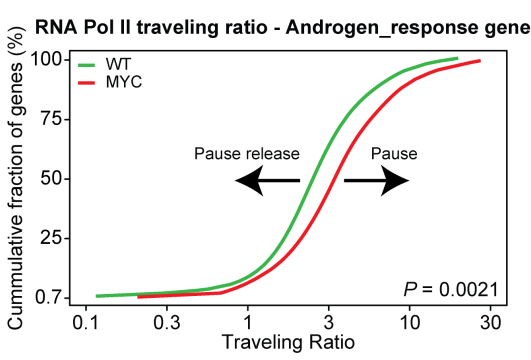

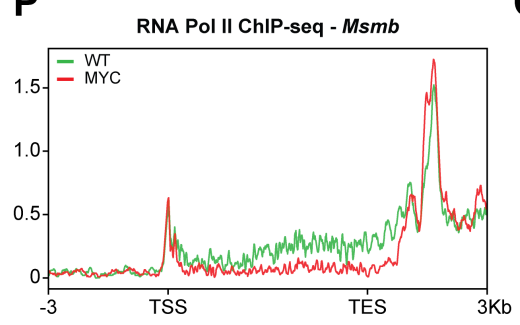

K GSEA - Pause release genes in MYC MYC_targets_V1
TNFA_signaling_via_NFKB p53_pathway Estrogen_response_arly Unfolded_protein_response KRAS_signoling_uP Estrogen_response_late ithelial_mesenchymal_transition Allograft_rejection. $-\log 10$ (FDR)
GSEA - Pause genes in MYC

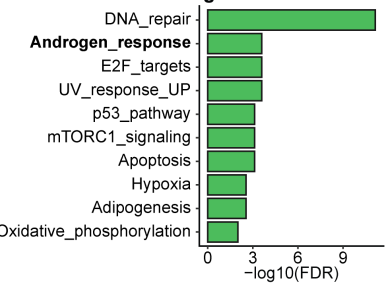
gen_response E2F_targets 每

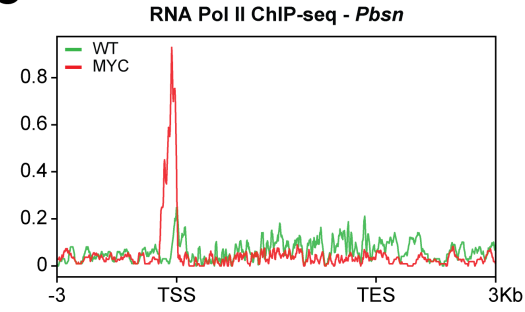

Figure 7 
967 Figure 7: MYC overexpression disrupts the AR transcriptional program by pausing AR 968 regulated genes. (A) BETA analysis revealed that AR binding sites are associated with gene 969 downregulation following MYC overexpression. (B, C) Despite a dampened AR transcriptional 970 program, higher levels of the AR binding (B) and H3K27ac mark (C) are observed nearby AR 971 response genes. (D-F) AR, FOXA1 and H3K27ac tracks at Pbsn locus, an AR-dependent gene, 972 reveal unchanged or heightened AR and FOXA1 binding (D) albeit decreased protein (E) and 973 transcript levels (F) following MYC overexpression. $(\mathbf{G}, \mathbf{H})$ Unchanged AR and FOXA1 binding 974 and H3K27ac mark at Mmsb locus (G), an AR-dependent gene downregulated by MYC 975 overexpression (H). (I) RNA Pol II traveling ratio differences following MYC overexpression in 976 murine VP. (J, K) Pause release genes following MYC overexpression are characterized by greater 977 RNA Pol II occupancy at gene body $(\mathbf{J})$ and are enriched for MYC transcriptional signatures 978 (GSEA, Hallmark, $\mathrm{P}<0.05$ and FDR $<0.1$; K). (L, M) Pause genes following MYC overexpression 979 are characterized by greater promoter-proximal RNA Pol II occupancy (L) and are enriched for 980 AR transcriptional signature (GSEA, Hallmark, $\mathrm{P}<0.05$ and $\mathrm{FDR}<0.1 ; \mathbf{M})$. (O, $\mathbf{P})$ Increased RNA 981 Pol II occupancy at the promoter of Pbsn (O) and decreased occupancy at the gene body of Msmb 982 (P) following MYC overexpression. (Q) RNA Pol II traveling ratio reveals greater promoter983 proximal pausing at Androgen_response genes. 
bioRxiv preprint doi: https://doi.org/10.1101/2021.04.23.441016; this version posted April 24, 2021. The copyright holder for this preprint (which was not certified by peer review) is the author/funder, who has granted bioRxiv a license to display the preprint in perpetuity. It is made available under aCC-BY 4.0 International license.

984
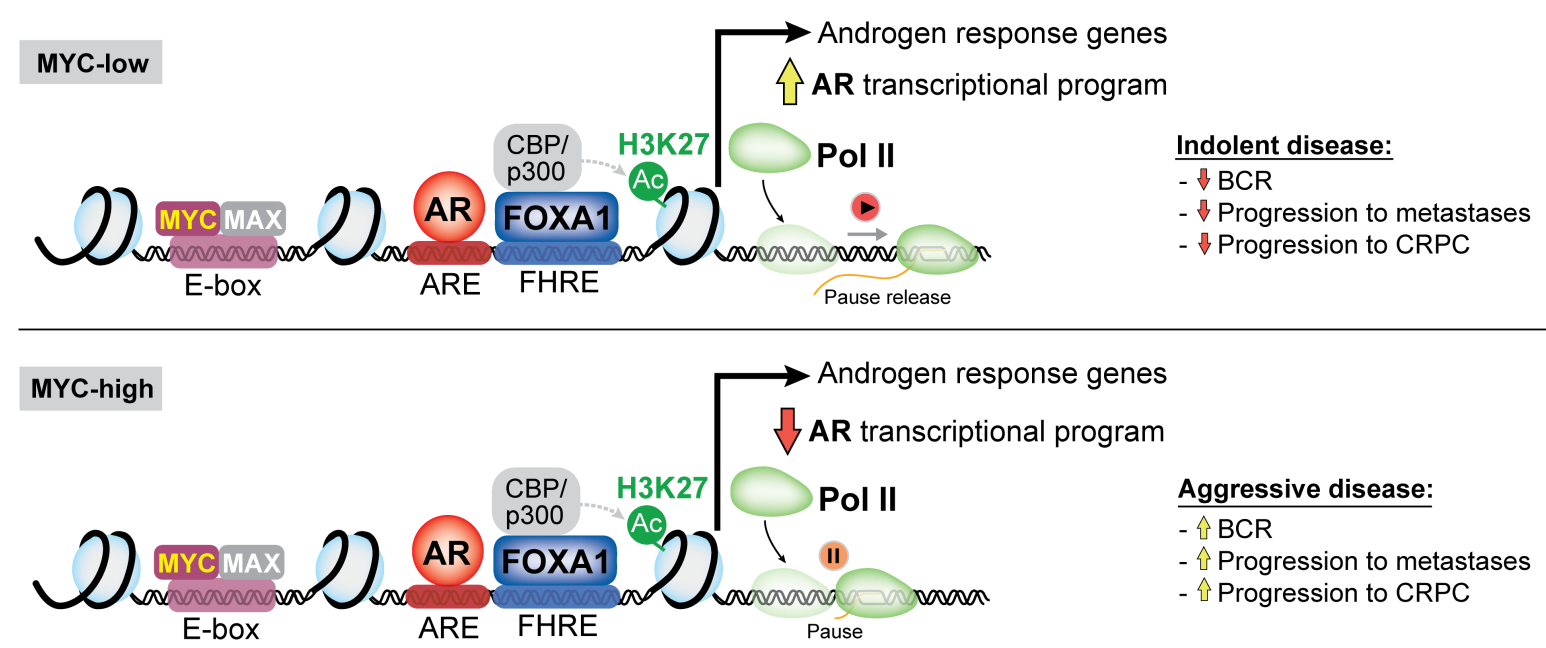

\section{Graphical summary.}


987 SUPPLEMENTARY FIGURES

A
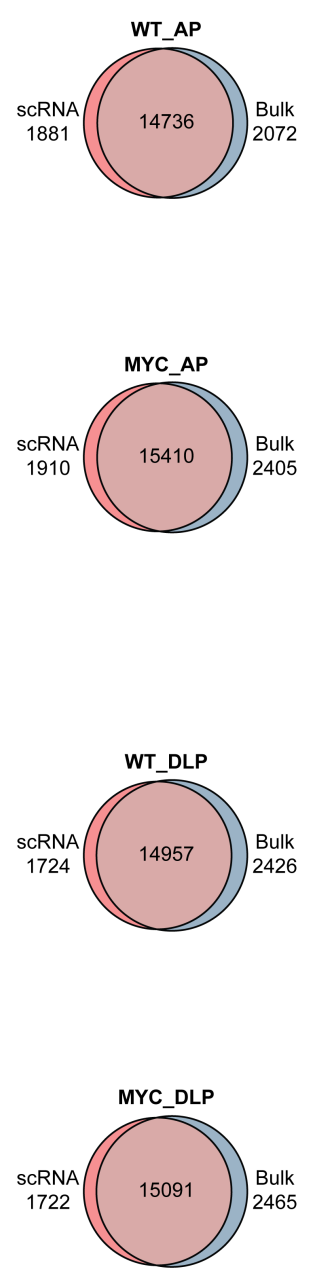
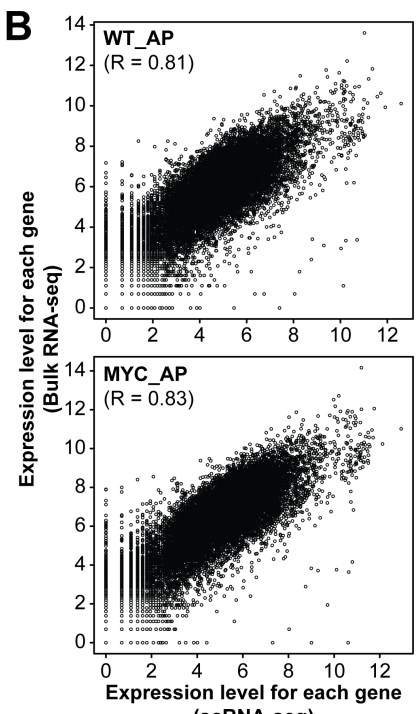

(scRNA-seq) DLP lobes. (A, B) Transcriptional profiling of WT and MYC-transformed AP and DLP lobes reveals high concordance for the total number of genes detected (A) and their expression levels (B) prostate. 
A

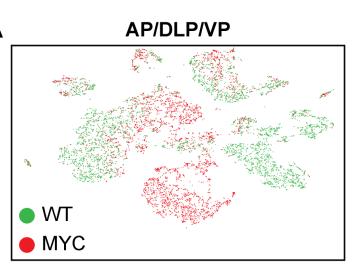

\section{$\mathbf{B}_{\text {Basal }}$}

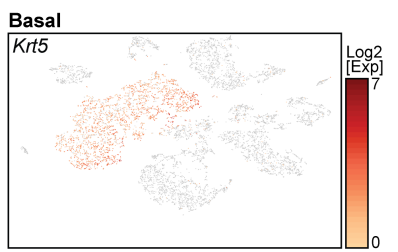

Basal

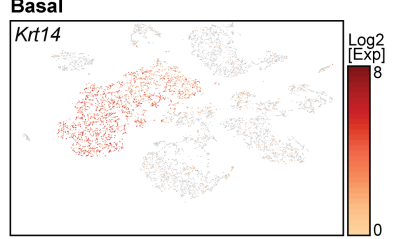

Progenitor

Psca

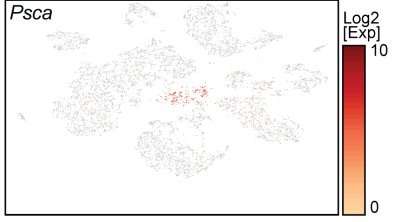

Progenitor

Cd44

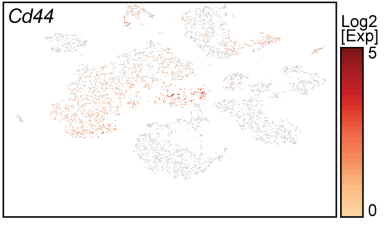

Progenitor

Tacstd2

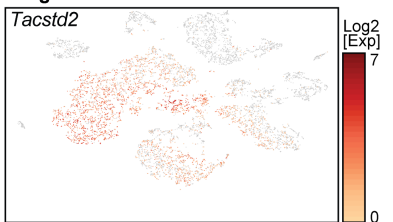

AP
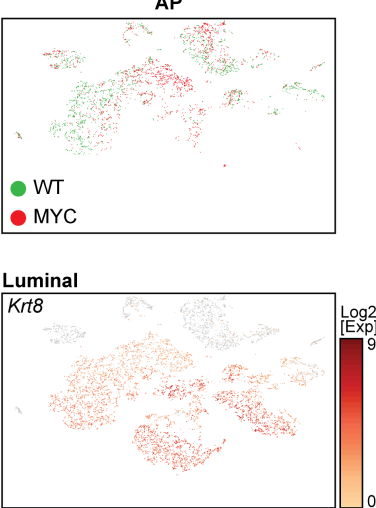

Luminal

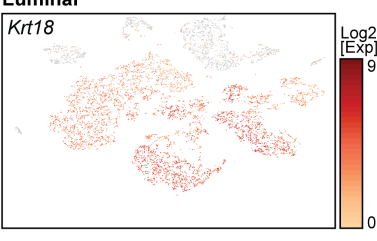

Mesenchymal

Wnt2

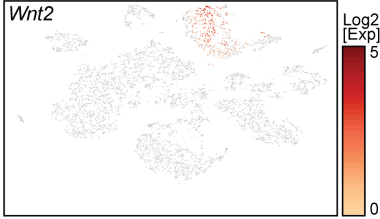

Mesenchymal

Pdgfra

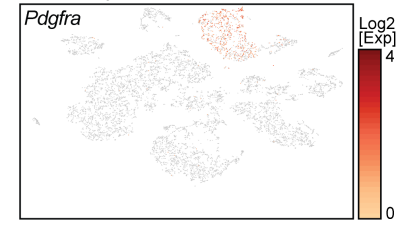

Erythrocyte

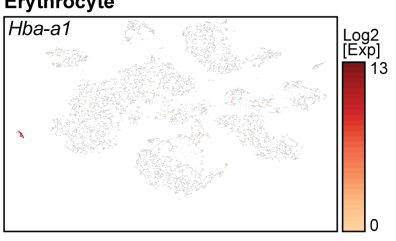

DLP

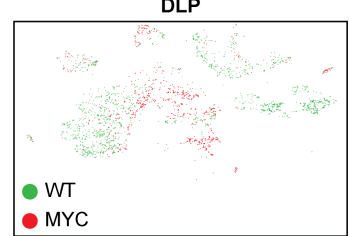

T cell

Cd3d

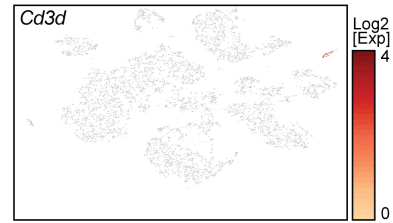

Macrophage / monocyte

Cd14

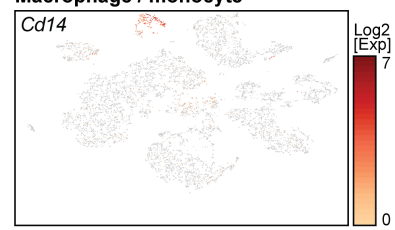

Smooth muscle

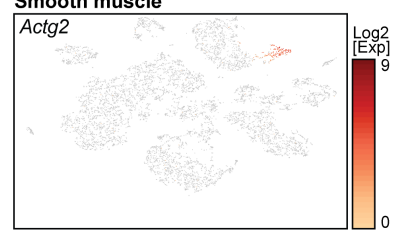

Vascular endothelium

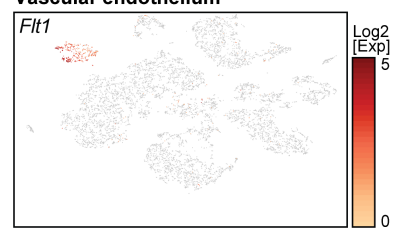

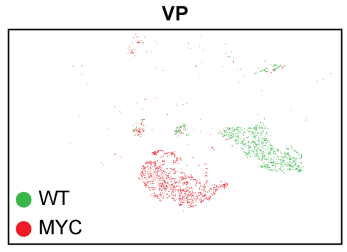

$C_{\text {MYC-expressing cell }}$

mm10Myc

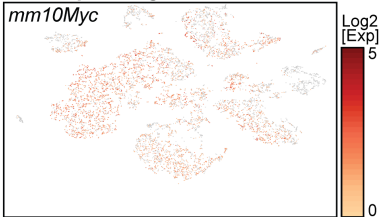

MYC-expressing cell

hg19MYC

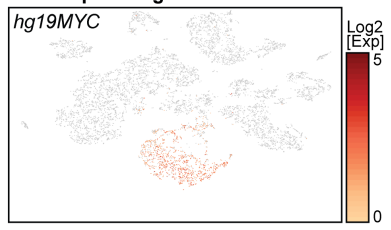

AR-expressing cell

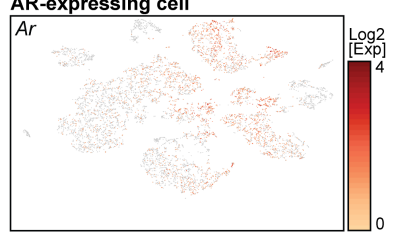

Figure S2: Molecular characterization of murine WT and MYC-transformed prostate lobes. (A) Single-cell census of WT and MYC-transformed AP, DLP and VP. tSNE of scRNA-seq profiles (as in Figure 2A), colored by genotype. (B, C) Expression of selected markers of different subsets (B) as well as murine $M y c(m m 10 M y c)$, human MYC $(h g 19 M Y C)$ and the $\operatorname{Ar}(\mathbf{C})$. WT: wildtype; VP: ventral prostate; DLP: dorsolateral prostate; AP: anterior prostate. 
bioRxiv preprint doi: https://doi.org/10.1101/2021.04.23.441016; this version posted April 24, 2021. The copyright holder for this preprint (which was not certified by peer review) is the author/funder, who has granted bioRxiv a license to display the preprint in perpetuity. It is made available under aCC-BY 4.0 International license.

1000

1001

1002

1003

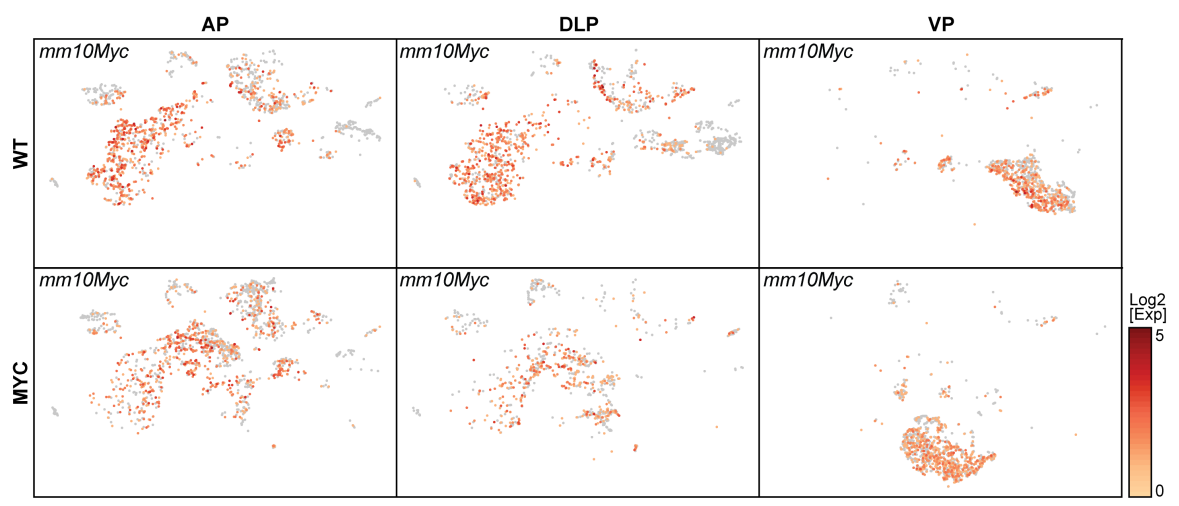

Figure S3: Murine $M y c$ is expressed across cell subpopulations and prostate lobes. Expression of murine $M y c(m m 10 M y c)$ in WT and MYC-transformed AP, DLP and VP. WT: wild-type; AP: anterior prostate; DLP: dorsolateral prostate; VP: ventral prostate. 
bioRxiv preprint doi: https://doi.org/10.1101/2021.04.23.441016; this version posted April 24, 2021. The copyright holder for this preprint (which was not certified by peer review) is the author/funder, who has granted bioRxiv a license to display the preprint in perpetuity. It is made available under aCC-BY 4.0 International license.

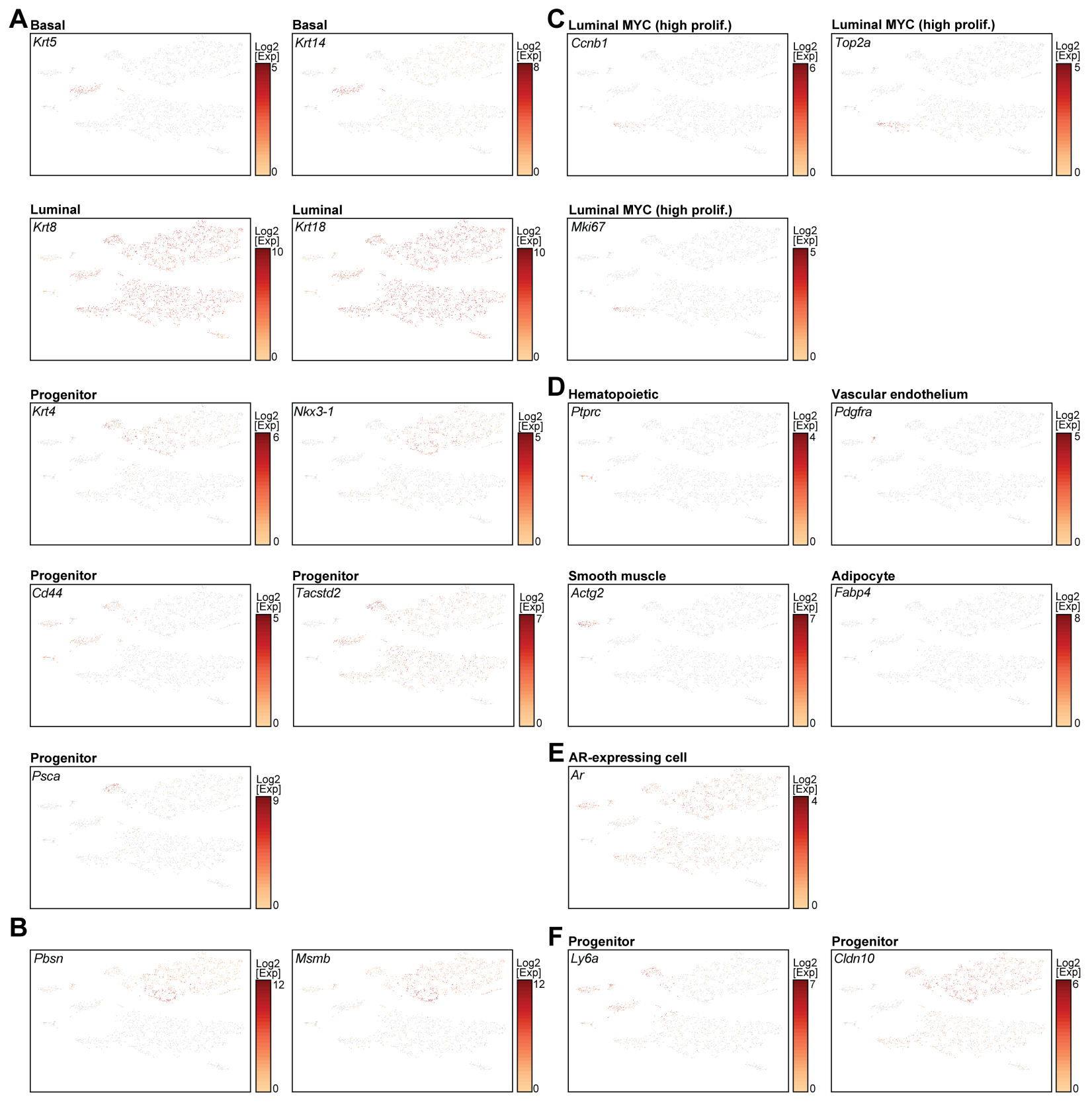

Figure S4: Molecular characterization of murine WT and MYC-transformed VP. (A-F) Expression of selected markers of different subsets. WT: wild-type; VP: ventral prostate. 
bioRxiv preprint doi: https://doi.org/10.1101/2021.04.23.441016; this version posted April 24, 2021. The copyright holder for this preprint (which was not certified by peer review) is the author/funder, who has granted bioRxiv a license to display the preprint in perpetuity. It is made available under aCC-BY 4.0 International license.
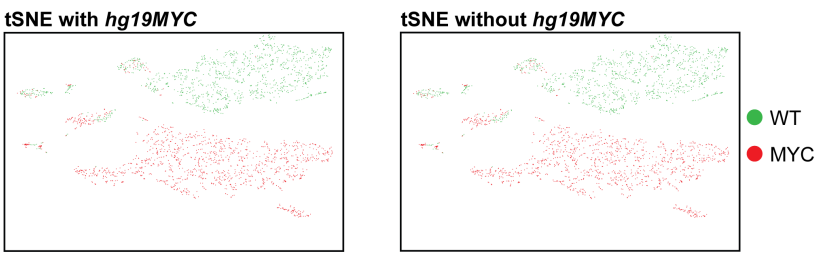

1008 Figure S5: tSNE of scRNA-seq profiles is not affected by the inclusion of human MYC 1009 transcript. tSNE of VP generated with (left) or without (right) the inclusion of human MYC 1010 (hg19MYC). WT: wild-type; VP: ventral prostate. 


\section{A}

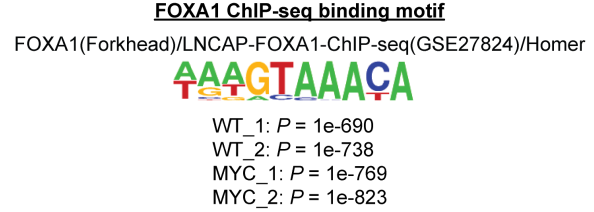

B Luminal subpopulation

- Luminal 1

- Luminal 2

- Luminal MYC

-Luminal MYC

ㄱ $7^{\text {(high prolif.) }}$

क्ष

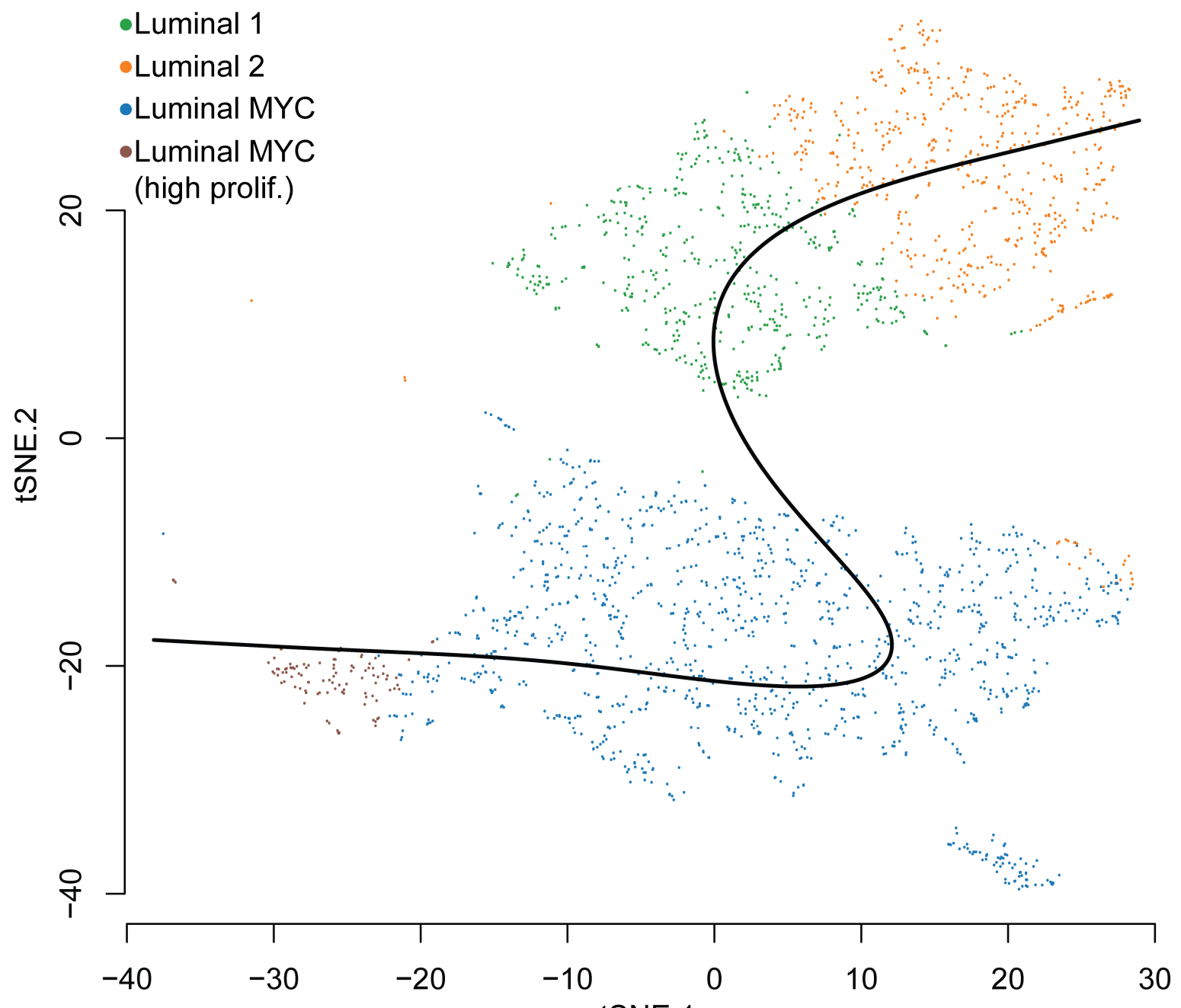

1012 Figure S6: Integration of ChIP-seq with scRNA-seq. (A) FOXA1 ChIP-seq identifies FHRE as 1013 the top FOXA1 binding motif in WT and MYC-transformed VP. (B) Slingshot pseudotime 1014 inference used to order luminal cells in Figure 4G. WT: wild-type; VP: ventral prostate; FHRE: 1015 forkhead response element. 


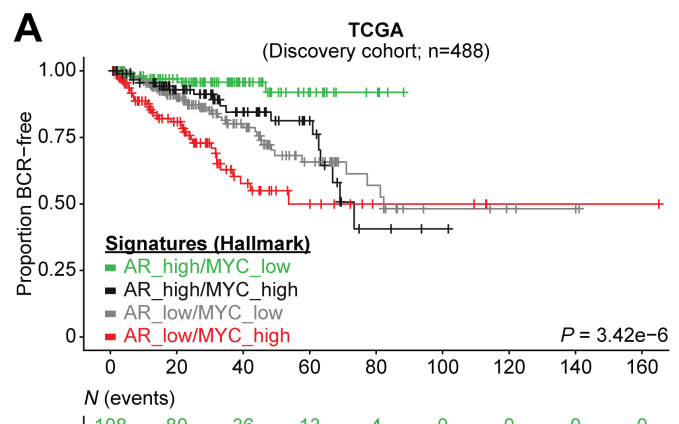

B signatures AR_low/ AR_low/ AR_high/ AR_high/
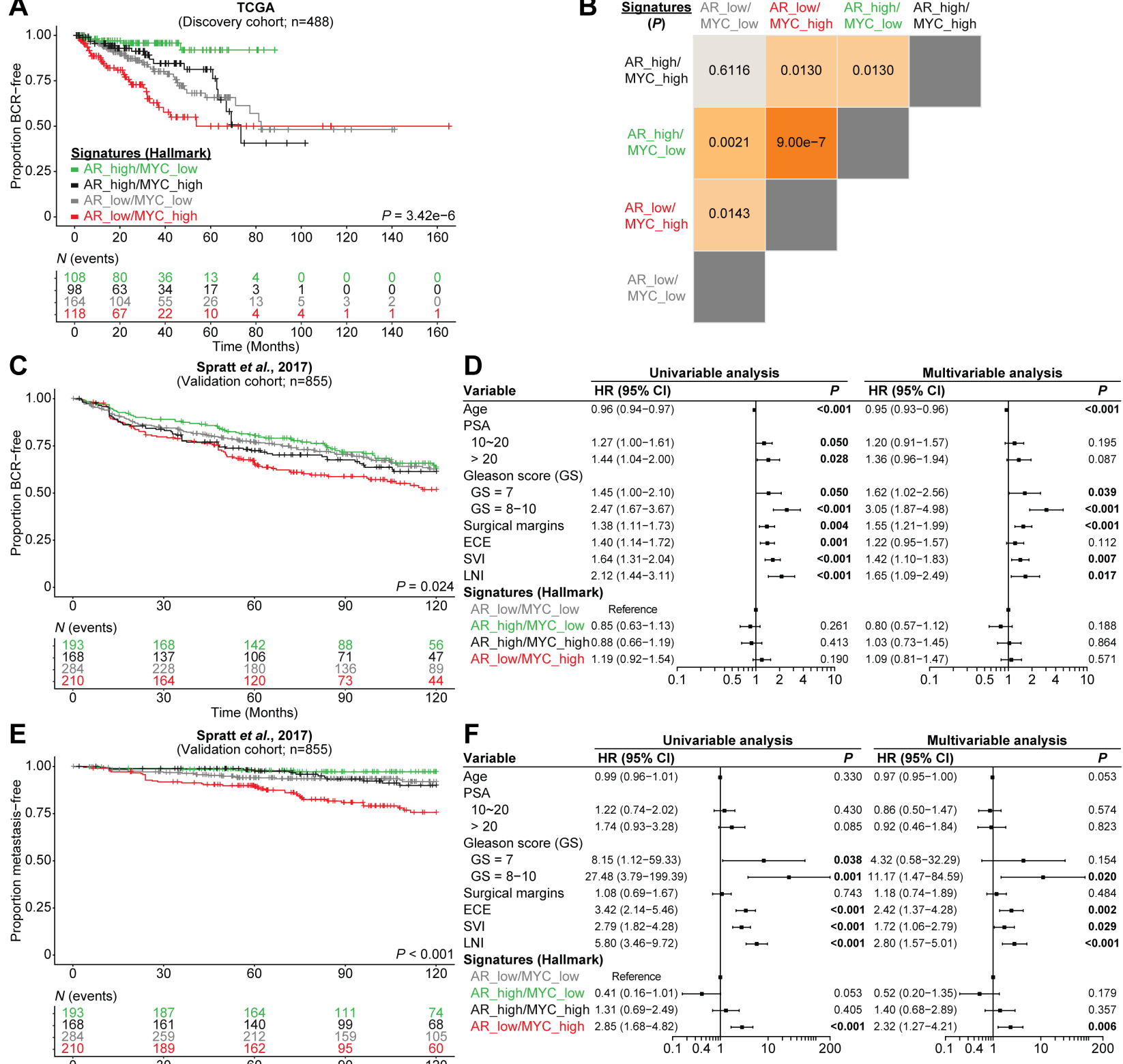

Figure S7: Divergent MYC and AR transcriptional programs dictate disease progression. (A, B) Kaplan-Meier curves (A) and log-rank tests (B) reveal that patients bearing a primary tumor characterized by low AR transcriptional signature (Hallmark) and concurrent high MYC transcriptional signature (Hallmark) have a shorter time to biochemical recurrence (BCR) within the discovery cohort (TCGA). (C, D) Kaplan-Meier curves (C) but not univariable and multivariable analysis (D) confirms that tumors with concurrent low AR and high MYC transcriptional signatures have a significant more rapid development of BCR than tumors with low AR transcriptional signature without an active MYC transcriptional program in the validation cohort (Spratt et al., 2017). (E, F) Kaplan-Meier curves (E), univariable and multivariable analyses (F) reveal that tumors with concurrent low AR and high MYC transcriptional signatures are more likely to develop metastatic disease. PSA: prostate-specific antigen; HR: hazard ratio; GS: Gleason score; ECE: extracapsular extension; SVI: seminal vesicles invasion; LNI: lymph node involvement. 
A
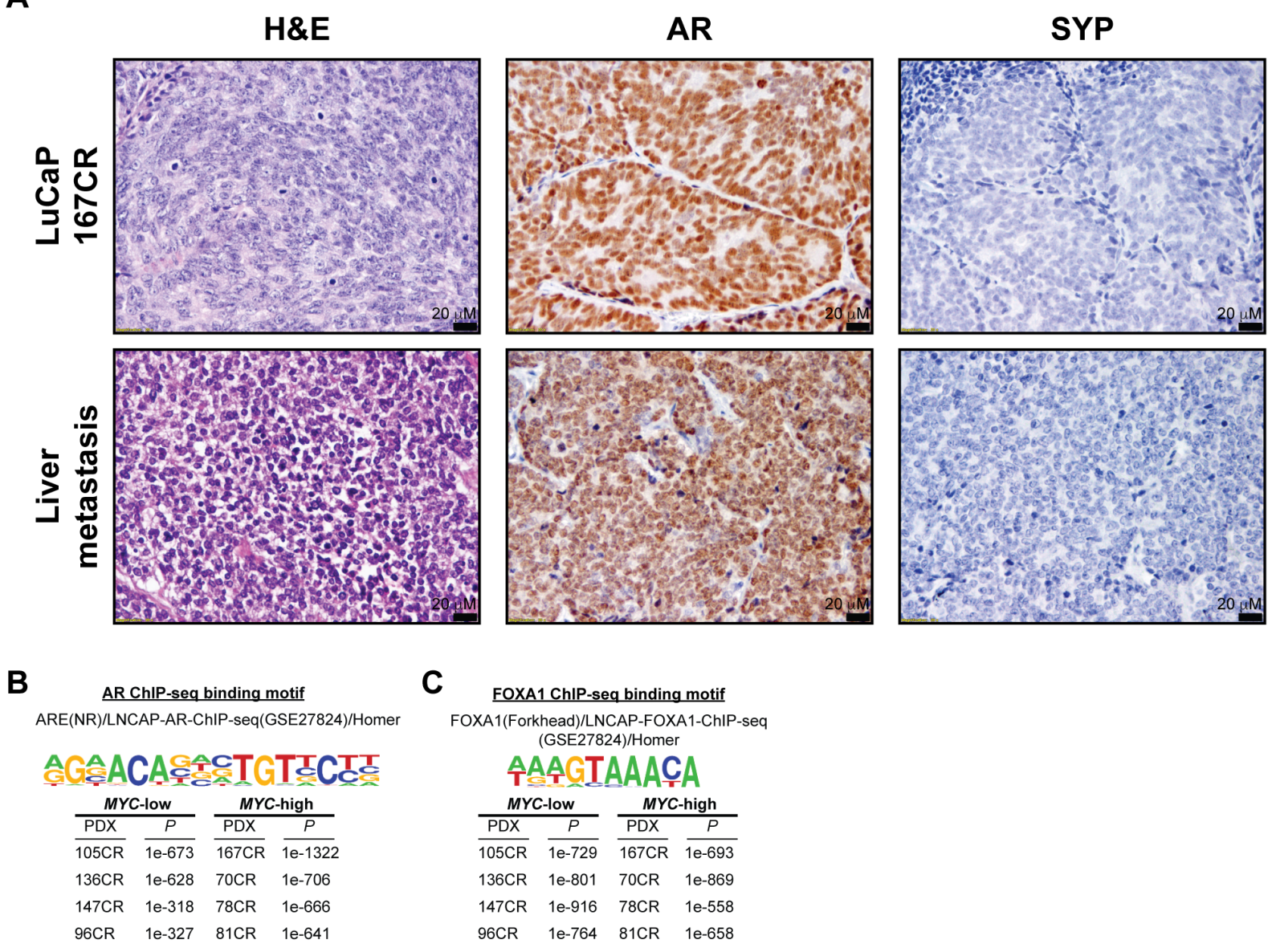

Figure S8: mCRPC LuCaP patient-derived xenograft (PDX) 167CR characterization and top binding motifs in LuCaP PDXs AR and FOXA1 ChIP-seq. (A) LuCaP PDX 167CR was established from a liver metastasis of 77-year old Caucasian male who died of abiraterone-, carboplatin- and docetaxel-resistant CRPC. LuCaP 167CR expresses AR, responds to castration and is negative for synaptophysin. Morphology of the PDX was concordant with the original liver metastasis (B, C) AR and FOXA1 ChIP-seq identifies ARE (B) and FHRE $(\mathbf{C})$, respectively as the top binding motif in mCRPC LuCaP PDXs. ARE: androgen response element; FHRE: forkhead response element. 
bioRxiv preprint doi: https://doi.org/10.1101/2021.04.23.441016; this version posted April 24, 2021. The copyright holder for this preprint (which was not certified by peer review) is the author/funder, who has granted bioRxiv a license to display the preprint in perpetuity. It is made available under aCC-BY 4.0 International license.

A
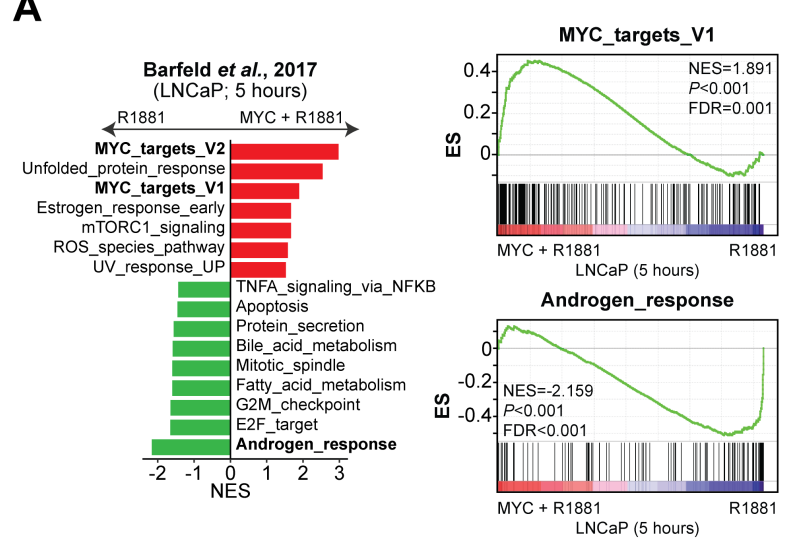

B

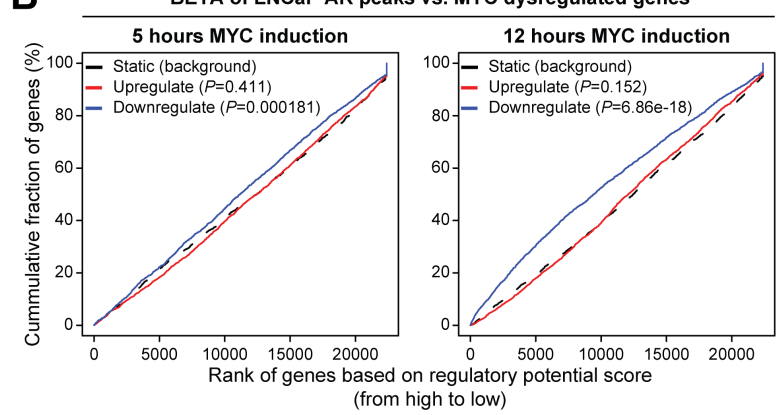

C

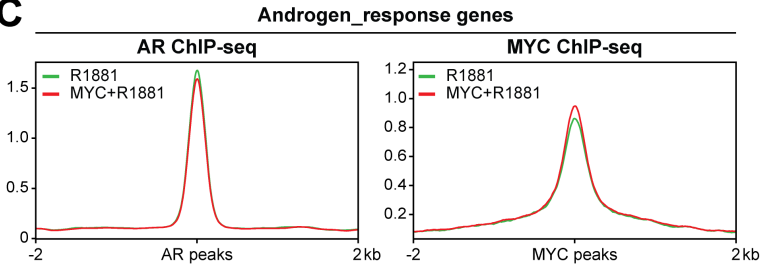

D
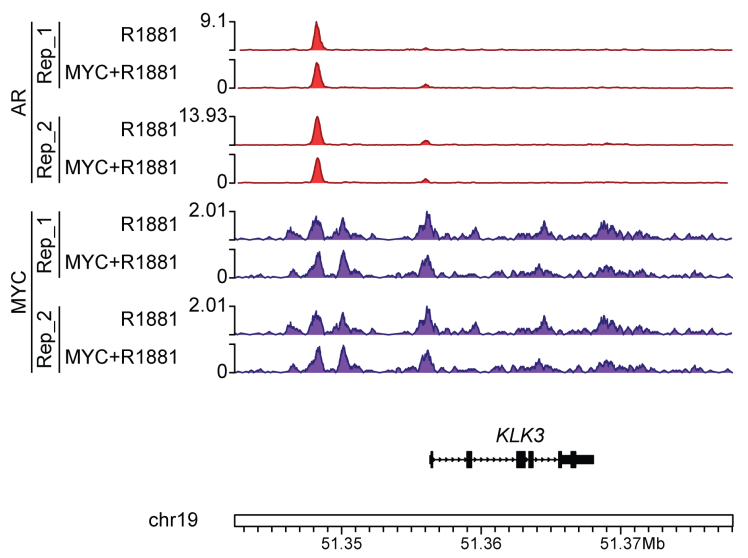
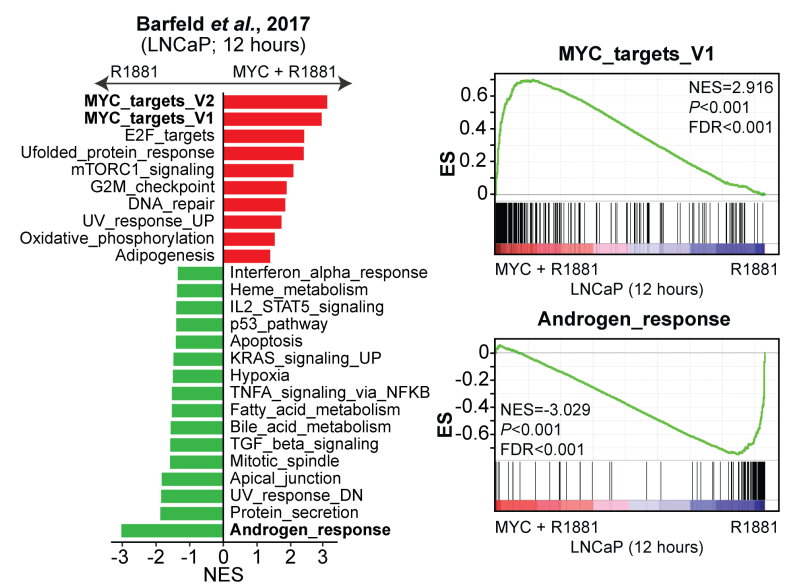

BETA of LNCaP MYC peaks vs. MYC dysregulated genes

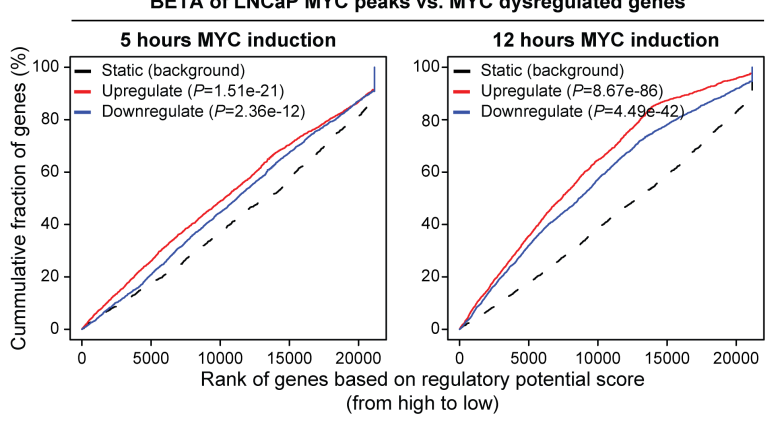

MYC_targets_V1 genes
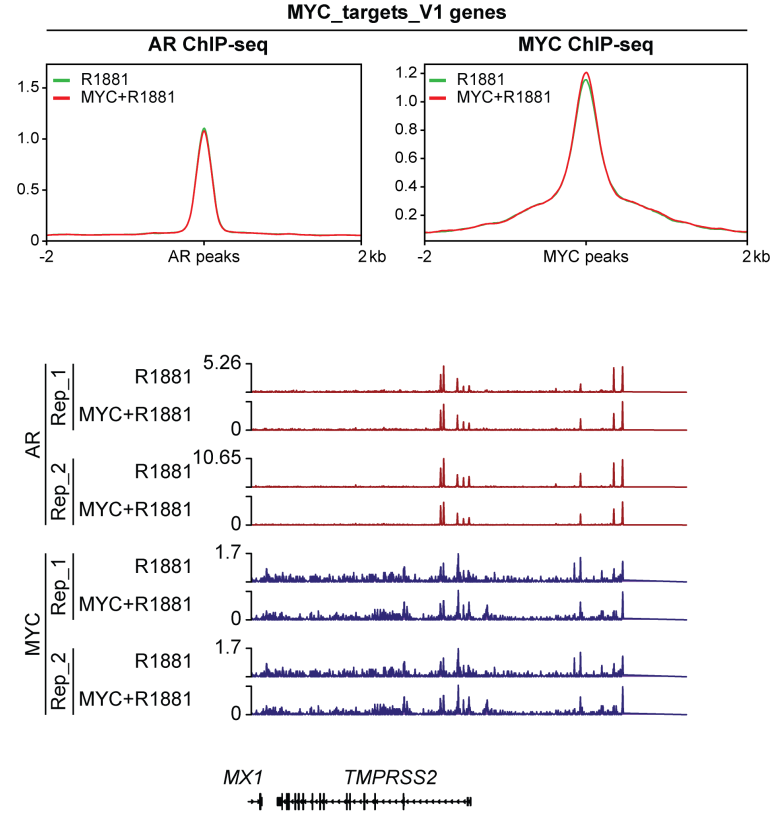

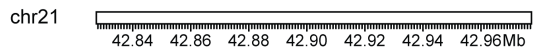


1040 Figure S9: MYC overexpression disrupts the AR transcriptional program in LNCaP cells. 1041 Reanalysis of transcriptomics and epigenetics data from Barfed and colleagues ${ }^{15}$. (A) Gene Set 1042 Enrichment Analysis (GSEA, Hallmark, $\mathrm{P}<0.05$ and FDR $<0.1$ ) revealed an enriched MYC 1043 transcriptional program and a depleted AR response following 5 or 12 hours of MYC induction. 1044 (B) BETA analysis revealed that AR binding sites are associated with gene downregulation while 1045 MYC binding sites are associated with gene upregulation following MYC induction. (C) AR and 1046 MYC binding nearby Androgen_response and MYC_targets_V1 genes is unchanged following 1047 MYC induction despite a dampened AR and a heightened MYC transcriptional program. (D) 1048 Unchanged AR and MYC binding at KLK3 and TMPRSS2 loci, AR-dependent genes 1049 downregulated by MYC overexpression. 
bioRxiv preprint doi: https://doi.org/10.1101/2021.04.23.441016; this version posted April 24, 2021. The copyright holder for this preprint (which was not certified by peer review) is the author/funder, who has granted bioRxiv a license to display the preprint in perpetuity. It is made available under aCC-BY 4.0 International license.

A

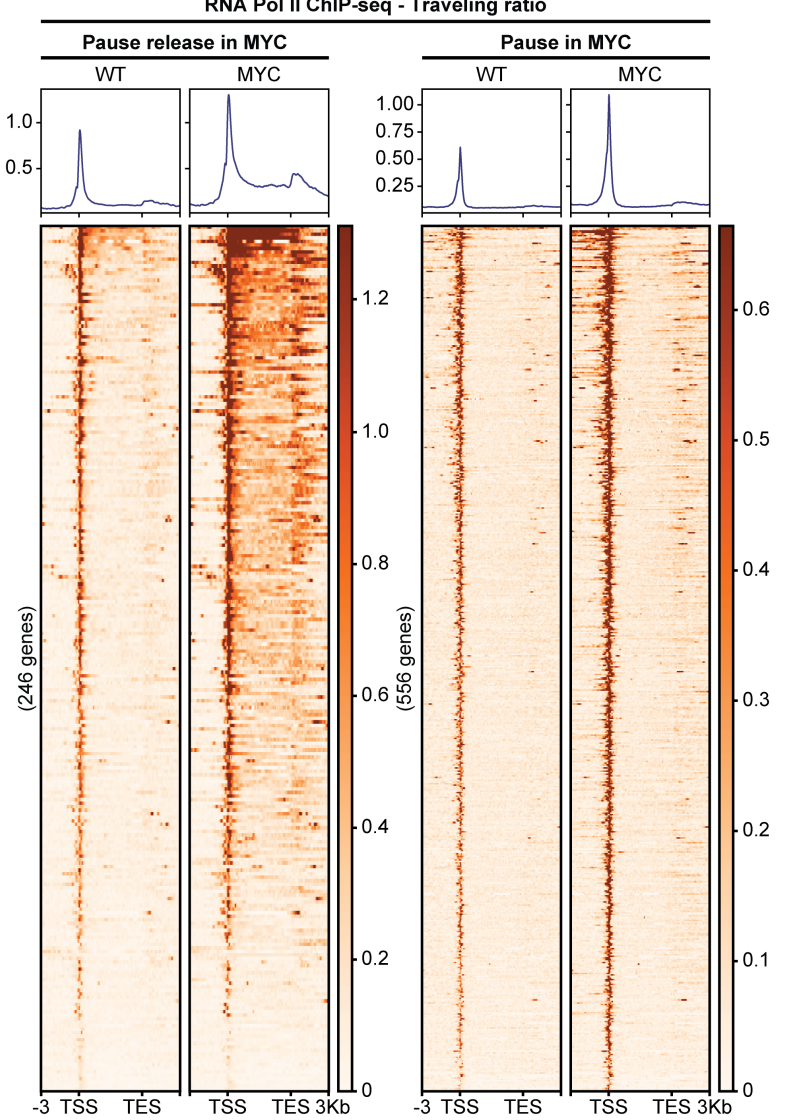

B

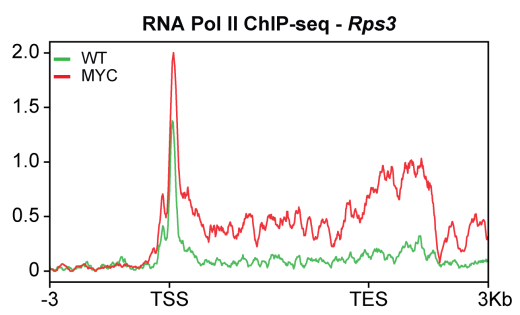

C

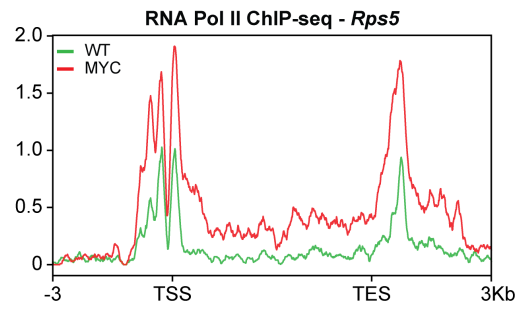

D

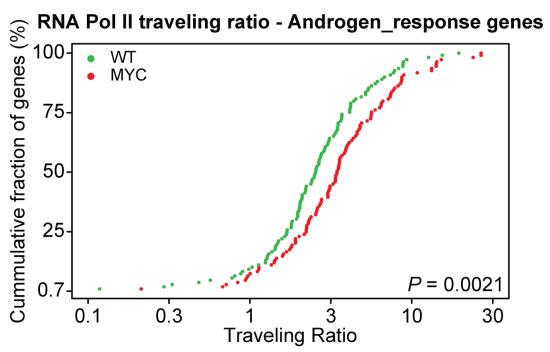

1051 Figure S10: RNA Pol II promoter-proximal pausing. (A) RNA Pol II occupancy at pause release Rps6 (C) MYC_targets_V1 genes. (D) RNA Pol II traveling ratio reveals greater promoterproximal pausing at Androgen_response genes (non-smoothed curves). 


\section{Composite}

AR
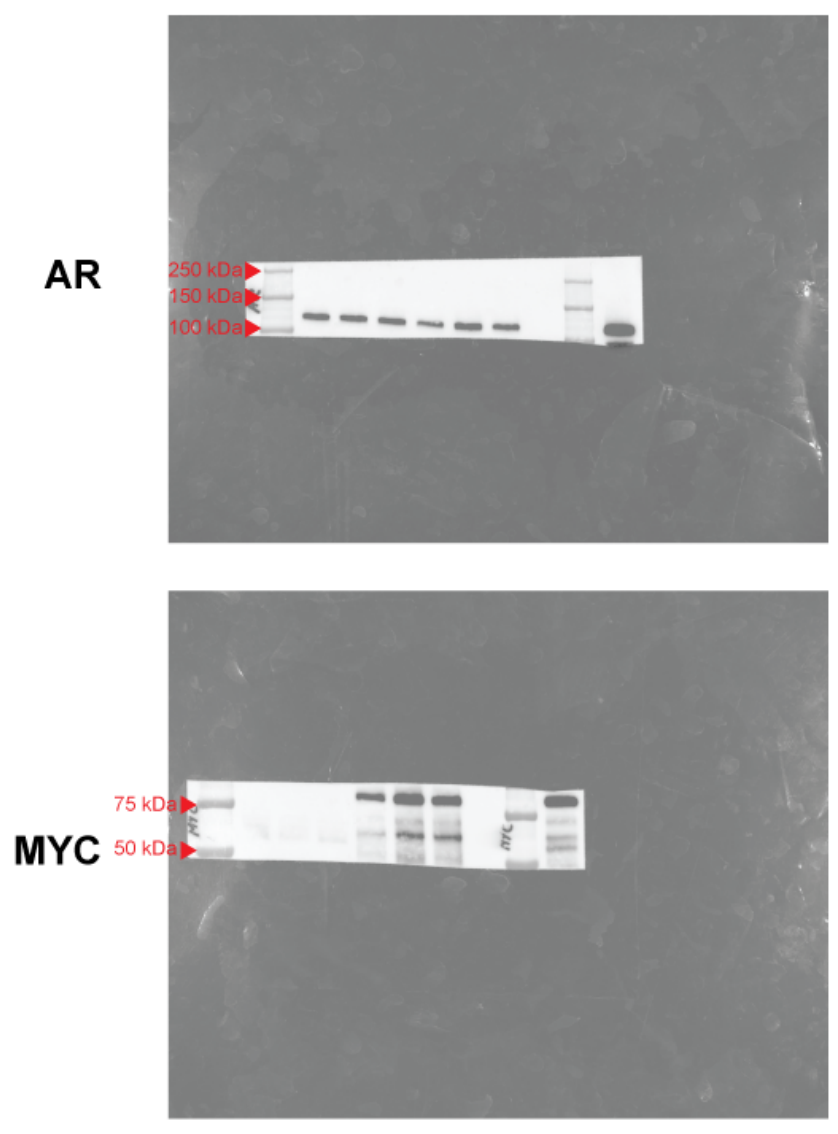

$\beta$-Actin

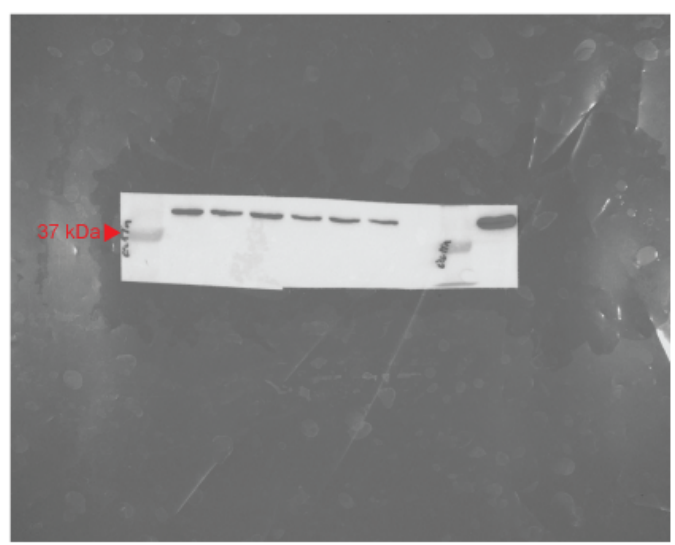

Chemilumininescence
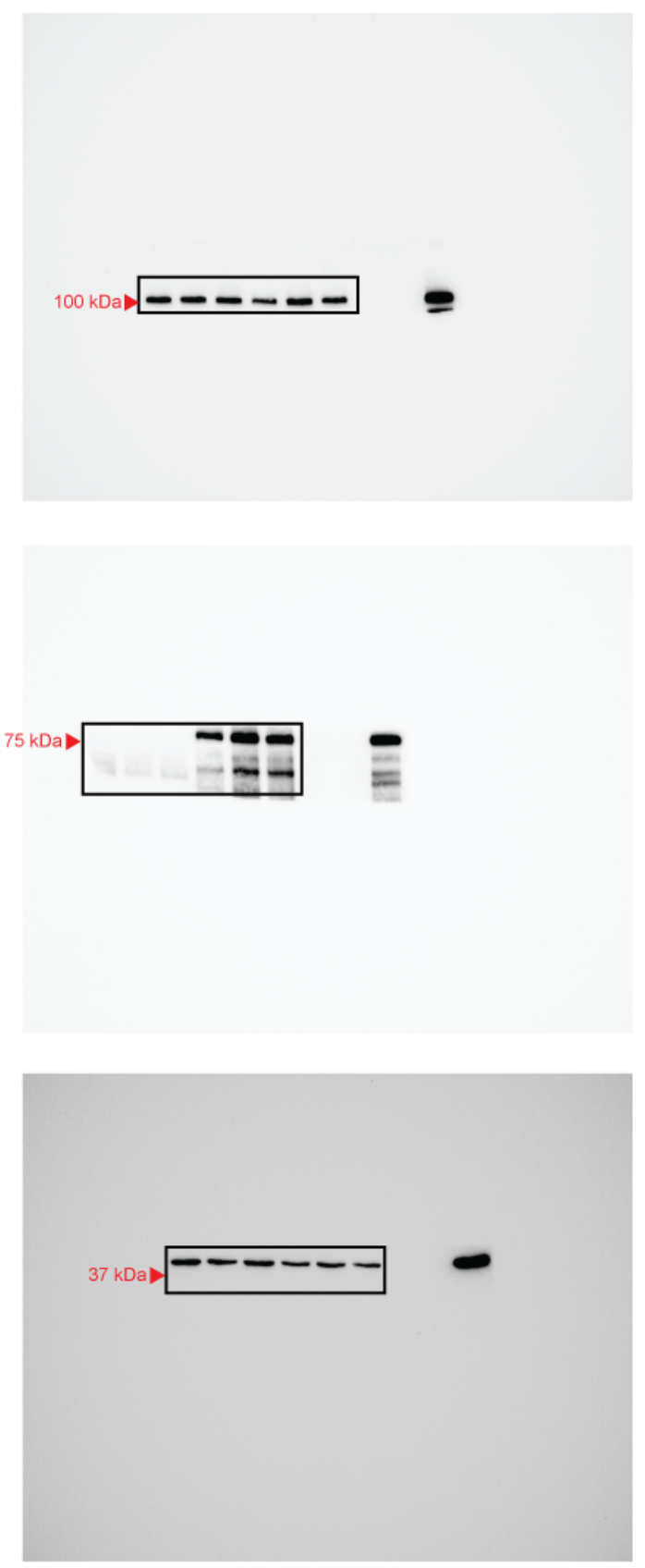

1057 Supplementary Data 1: Unprocessed western blots from Figure 3E. 
bioRxiv preprint doi: https://doi.org/10.1101/2021.04.23.441016; this version posted April 24, 2021. The copyright holder for this preprint (which was not certified by peer review) is the author/funder, who has granted bioRxiv a license to display the preprint in perpetuity. It is made available under aCC-BY 4.0 International license.
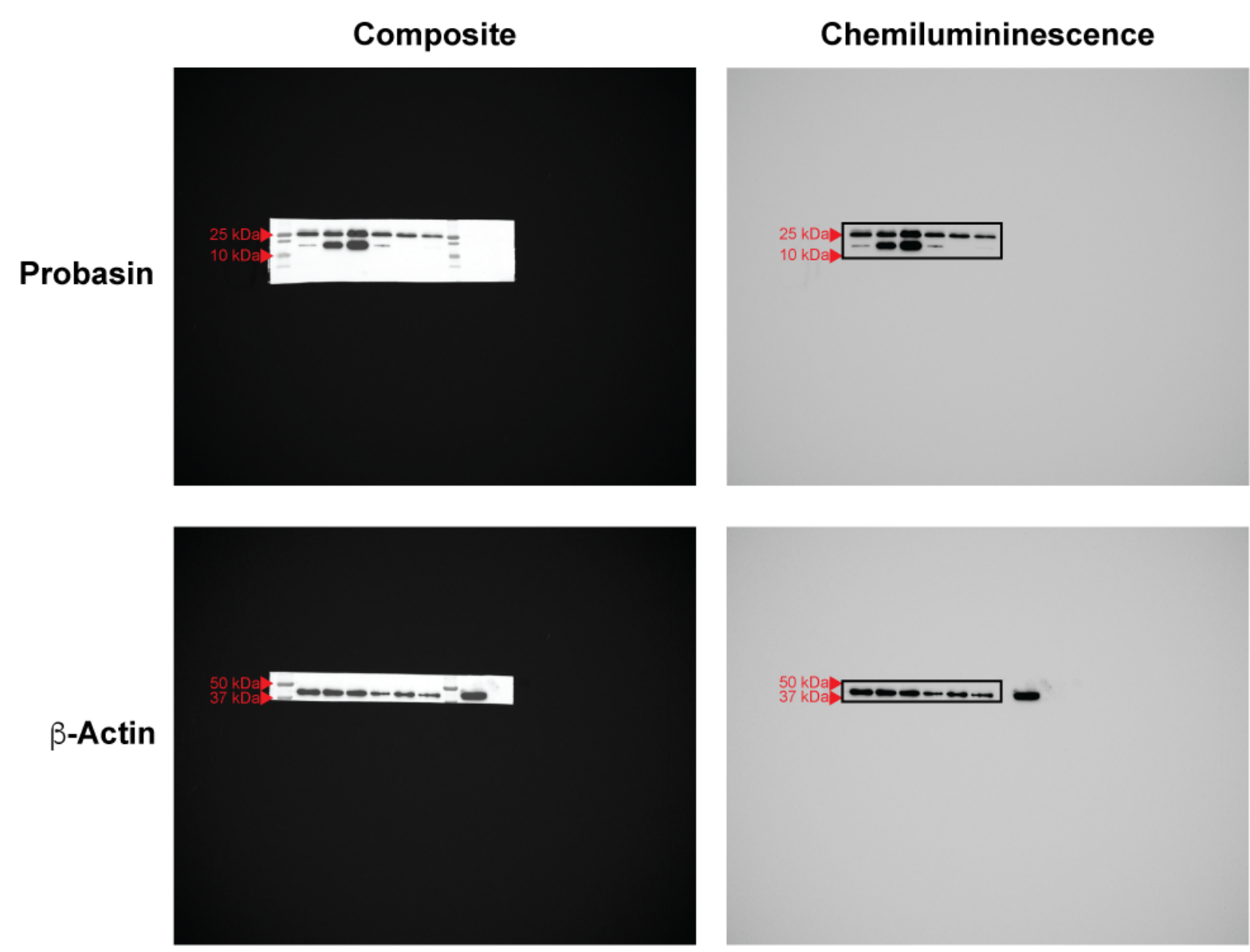

1059 Supplementary Data 2: Unprocessed western blots from Figure 7E. 